\title{
Scaling of rotational inertia of primate mandibles
}

Callum F. Ross ${ }^{a},{ }^{*}$, Jose Iriarte-Diaz ${ }^{b}$, Ellen Platts ${ }^{c}$, Treva Walsh ${ }^{c}$, Liam Heins ${ }^{c}$, Geoffrey E. Gerstner ${ }^{\mathrm{d}}$, Andrea B. Taylor ${ }^{\mathrm{e}}$

${ }^{a}$ Department of Organismal Biology and Anatomy, The University of Chicago

${ }^{b}$ Department of Oral Biology, College of Dentistry, University of Illinois at Chicago

${ }^{\mathrm{C}}$ The College, The University of Chicago

${ }^{\mathrm{d} D e p a r t m e n t}$ of Biologic and Materials Sciences, School of Dentistry, University of Michigan

${ }^{e}$ Department of Basic Science, Touro University California

${ }^{*}$ Corresponding author:

E-mail address: rossc@uchicago.edu (C.F. Ross)

Keywords: Mastication, Biomechanics, Chewing frequency, Kinematics, Neural control

C 2017. This manuscript version is made available under the Elsevier user license 


\section{Abstract}

The relative importance of pendulum mechanics and muscle mechanics in chewing dynamics has implications for understanding the optimality criteria driving the evolution of primate feeding systems. The Spring Model (Ross et al., 2009b), which modeled the primate chewing system as a forced mass-spring system, predicted that chew cycle time would increase faster than was actually observed. We hypothesized that if mandibular momentum plays an important role in chewing dynamics, more accurate estimates of the rotational inertia of the mandible would improve the accuracy with which the Spring Model predicts the scaling of primate chew cycle period. However, if mass-related momentum effects are of negligible importance in the scaling of primate chew cycle period, this hypothesis would be falsified. We also predicted that the greater mandibular "robusticity" of anthropoid mandibles compared with prosimians would be associated with higher moments of inertia for anthropoid mandibles. From computed tomography scans, we estimated the scaling of the moment of inertia $\left(I_{j}\right)$ of the mandibles of thirty-one species of primates, including 22 anthropoid and nine prosimian species, separating $l_{j}$ into the moment about a transverse axis through the center of mass $\left(I_{x x}\right)$ and the moment of the center of mass about plausible axes of rotation. We found that across primates $l_{j}$ increases with positive allometry relative to jaw length, primarily due to positive allometry of jaw mass and $I_{x x}$, and that anthropoid mandibles have greater rotational inertia compared with prosimian mandibles of similar length. Positive allometry of $l_{j}$ of primate mandibles actually lowers the predictive ability of the Spring Model, suggesting that scaling of primate chew cycle period, and chewing dynamics in general, are more strongly influenced by factors other than scaling of inertial properties of the mandible, such as the dynamic properties of the jaw muscles and neural control. Differences in cycle period scaling between chewing and locomotion systems reinforce the suggestion that displacement and force control are more important in the design of feeding systems than energetics and speed. 


\section{Introduction}

Investigations of the determinants and consequences of size-related changes (scaling) in the periodicity of cyclic movements provide important insights into the design (form-function relationships) of musculoskeletal systems (McMahon, 1975; Heglund and Taylor, 1988; Biewener, 2005; Moore et al., 2010; Norberg and Aldrin, 2010; Hooper, 2012; Kilbourne and Hoffman, 2013a). Much of this work has focused on the scaling of step cycle period in locomotor systems (Cavagna et al., 1997; de Ruiter et al., 2013; Marsh et al., 2004; Raichlen, 2004; Modica and Kram, 2005; Pontzer, 2007), reflecting the large proportion of overall energy budgets expended on locomotion (McNab, 2002), the large proportions of locomotor energetics spent on the internal work of relative movement of limbs and trunk (Pontzer, 2007), and the importance of locomotion for foraging and predator avoidance (Reilly et al., 2007). In comparison with studies of the locomotor system, less attention has been paid to the scaling of cycle periods in the chewing system (Fortelius, 1985; Druzinsky, 1993; Gerstner and Gerstein, 2008). The chewing system provides an interesting contrast with the locomotor system because its smaller size means that the overall energy costs of chewing are much lower than those of locomotion, suggesting that in the feeding system optimizing energetic efficiency may be less important than optimizing control of force and displacement (Ross et al., 2009b). Here we use scaling analyses of the moment of inertia of primate mandibles - the resistance of the mandible to elevation and depression rotations during chewing - to argue that primate chew cycle period is more strongly influenced by factors such as the dynamic properties of the jaw muscles and neural control than by mandibular inertial properties. In addition, we argue that differences in cycle period scaling between chewing and locomotion systems reinforce the suggestion that displacement and force control are more important in the design of feeding systems than energetics and speed.

Scaling of mammal step cycle period 
The scaling relationships between limb length and oscillation frequency are often explained with reference to pendular mechanics (for reviews see [Kilbourne and Hoffman, 2013b, 2015; Kilbourne, 2014]). The cycle period of a simple pendulum $\left(T_{p}\right)^{1}$-a pendulum with all its mass $\left(M_{p}\right)$ concentrated at the center of mass (CoM) suspended at a distance $L_{p}$ from the axis of rotation by a massless rod-is related to $L_{p}$ and the acceleration due to gravity $(g)$ by Equation [1].

$$
\begin{aligned}
& T p=2 \pi M p L p 2 M p g L p \\
& \text { which simplifies to } T p=2 \pi L p g \\
& \text { or } T p=2 \pi L p g 1 / 2
\end{aligned}
$$

The numerator is either a torque representing the moment of inertia (resistance to rotation) of the pendulum $\left(I_{p}\right)$ or $L_{p}$, a variable proportional to $I_{p}$, and the denominator is the driving torque, in this case gravity acting on pendular mass. Exponents for scaling relationships between limb step cycle periods and limb lengths that are close to $1 / 2$ have often been interpreted as support for the importance of pendular mechanics in the dynamics of mammalian limbs (Pennycuick, 1975; Alexander, 2003). Importantly, however, an exponent of $1 / 2$ does not require that pendular motion be driven by gravity. Indeed, not all models of limb step cycle period scaling include gravity in the denominator. McMahon's (1975) influential estimate of the scaling of natural period of limb oscillations in galloping mammals excludes gravity driven angular momentum altogether in favor of the driving torques of the limb muscles:

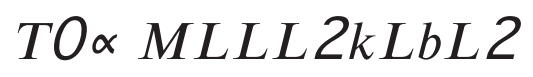

Here, the driving torque in the denominator consists of $k_{L}$, the spring constant of the limb muscles, acting about $b_{L}$, the limb muscle lever arm. The muscle spring constant $k_{L}$ is equal to $A_{p c s a} E / L_{m}$, where $A_{p c s a}$ is muscle physiological crosssectional area (PCSA), $L_{m}$ is muscle length, and $E$ is "the the slope of the

\footnotetext{
${ }^{1}$ Fractions are used to express scaling exponents predicted by theory and decimals to express exponents estimated empirically. A complete list of abbreviations and dimensionality of terms is presented in Table 1.
} 
stress/strain curve for the stimulated muscle" (McMahon, 1975). In support of modeling muscle properties with spring constants, the spring properties of muscles are widely acknowledged to confer not only energetic savings (McMahon, 1975; Lee and Farley, 1998), but also advantages for motor control of oscillating systems (Houk and Rymer, 1981; Nichols, 2002).

A richer understanding of the behavior of oscillating musculoskeletal systems emerges from simultaneous consideration of both gravity driven angular momentum and muscle properties in the same model. Turvey et al. (1988) presented a hybrid mass-spring/simple pendulum model, in which the numerator is again the moment of inertia of a simple pendulum and the denominator includes both a gravity-driven angular momentum torque, $M L g$, and a muscular spring-driven torque, $k b^{2}$ (Fig. 1). The natural period of this system is described by their "equation of the pendular clocking mode":

$$
T O=2 \pi M L 2 M L g+k b 2 .
$$

Turvey et al. (1988) hypothesized that the relative importance of angular momentum and muscle properties changes with system size: "as body mass decreases, the restoring moment relative to gravity becomes negligible in comparison with the restoring moment relative to the elasticity of the body's tissues" (311). Hooper (2012) later suggested that this explains why $T_{s}$ scales proportional to limb length $\left(L_{L}\right)$ as $T_{S} \alpha L_{L}{ }^{0.32}$ during trotting and $\alpha L_{L}{ }^{0.38}$ during galloping, instead of as $T_{s} \alpha L_{L}{ }^{0.5}$.

\section{Scaling of mammal chew cycle period}

In the feeding system, available data suggest that chew cycle duration $\left(T_{c}\right)$ scales proportional to jaw length $\left(L_{j}\right)$ with exponents similar to the exponent of $1 / 2$ predicted for a simple pendulum: across primates, $T_{c} \alpha L_{j}^{0.56}$ (Ross et al., 2009b) or $T_{c} \alpha L_{j}^{0.51}$ (Nagamine et al., 2013) and across a sample of herbivorous mammals, $T_{c} \alpha L_{j}^{055}$ (Shipley et al., 1994). Paradoxically, compared to limbs, oscillations of the mandible are less accurately described by pendular mechanics: mandibles are short compared with limbs and the architecture and 
neural control of chewing muscles arguably dampen mandibular oscillations during chewing (Wang and Stohler, 1991). These considerations led Ross et al. (2009b) to ask whether scaling of primate chew cycle period might be better explained by modeling the chewing system using a Spring Model derived from Turvey et al.'s (1988) hybrid mass-spring pendulum model. In implementing this "equation of the pendular clocking mode," Ross et al. (2009b) made assumptions that resulted in the equation converging on McMahon's model of limb mechanics (Equation [2] of the present paper; McMahon, 1975), which excludes gravity driven angular momentum altogether from the determinants of limb oscillation frequency. Ross et al. (2009b) estimated the scaling of jaw dry mass $\left(M_{j}\right)$, jaw length $\left(L_{j}\right)$, and elevator muscle lever arms $\left(b_{e}\right)$ from museum specimens and the scaling of jaw-elevator PCSAs from muscle architecture ( $A_{e}$; Anapol et al., 2008), and found that the Spring Model did not predict $T_{c}$ scaling as well as either a simple pendulum model or the best fit standardized major axis (SMA). Rather, the Spring Model predicted that chew cycle period should increase relative to $L_{j}$ with a steeper slope than it actually does. Why does the Spring Model predict scaling of primate chew cycle period with this bias?

\section{Improving the Spring Model}

In the original Spring Model, the moment of inertia of the jaw, $l_{j}$, was estimated as $M_{j} L_{j}^{2}$. This term will only scale proportional to $l_{j}$ if the distance from the center of mass (CoM) to the rotational axis is proportional to $L^{1.0}$ (Ross et al., 2009b), an assumption that could be violated by allometric and clade-related differences in mandibular size and shape. For example, strengthening of the mandibular symphysis in larger-bodied anthropoids (Ravosa, 1991) might cause the CoM to lie further from the axis of rotation than in prosimians, resulting in longer chewing cycle durations in anthropoids.

Our first hypothesis is that the Spring Mode/ will better predict the scaling of primate chewing frequencies when the rotational inertia of the mandible is accurately estimated. Specifically, this predicts that the moment of inertia of the mandible scales with negative allometry across primates, bringing the chew cycle 
periods predicted by the Spring Model more in line with the observed chew cycle periods. Our second, related hypothesis is that the greater mandibular robusticity (e.g., corpus and symphysis relative depth and width) of anthropoids compared with prosimians is associated with higher moments of inertia in anthropoids. This hypothesis predicts that, in the size range of primate mandibles, anthropoid moments of inertia scale relative to mandible length with higher intercepts than prosimians.

These two hypotheses assume that mandibular momentum plays an important role in chewing dynamics. However, the architecture and neural control of the jaw muscles have been argued to heavily dampen mandibular oscillations during chewing (Wang and Stohler, 1991; Ross et al., 2009b). If true, then we hypothesize that mass-related momentum effects are of negligible importance in the scaling of primate chew cycle period. This alternative to Hypothesis 1 predicts that accurate estimation of the moment of inertia of primate mandibles does not improve the performance of the Spring Model.

These three hypotheses are evaluated here using scaling exponents of moments of inertia of primate mandibles.

\section{Materials and methods}

\section{Samples}

We obtained computed tomography (CT) scans of 52 adult male and female mandibles from 31 species of primates, including nine prosimians and 22 anthropoids, and used these scans to estimate the location of the center of mass of the mandible, the distribution of mandibular mass relative to this center, and the location of the center of mass relative to four different assumed axes of rotation. Specimens used in this study are housed at the Field Museum of Natural History, Chicago, IL (FMNH), and the Smithsonian Institution, Washington, D.C. (SMNH; Table 2). All mandibles are complete and, in the case of the animals with unfused symphyses, still connected across the midline by dried symphyseal tissue. Specimen numbers are provided in the Supplementary 
Online Material (SOM) Table 1, along with notes on condition of the specimens (e.g., chipped or missing teeth).

Specimens from the FMNH (maximum diameter of $9 \mathrm{~cm}$ ) were scanned at the University of Chicago using a GMI-Triumph Tri-Modality Pre-Clinical micro $\mathrm{PET} / \mathrm{SPECT} / \mathrm{CT}$. The $\mathrm{x}$-ray source was set to $75 \mathrm{kVp}$ and 135 milliAmps. $360^{\circ}$ projection images (1184 x 1120 pixels) were acquired in a full circle trajectory, averaging five frames at each position. The projection data were reconstructed into a three dimensional image volume (512 x $512 \times 512$ voxels) using the system's filtered back-projection reconstruction algorithm at a general purpose setting. Slice thickness was $500 \mu \mathrm{m}$ or less. Hominoid SMNH specimens were scanned at the Smithsonian on a Siemens SOMATOM Emotion 6 CT scanner at $110 \mathrm{kV}, 70 \mathrm{~mA}, 1 \mathrm{~mm}$ slice thickness, $0.1 \mathrm{~mm}$ reconstruction increment, and a H50 moderately sharp kernel. The non-hominoid primates at the SMNH were scanned with a newer SIEMENS Somatom Emotion CT scanner at $110 \mathrm{kV}, 70$ $\mathrm{mA}, 0.6 \mathrm{~mm}$ slice thickness, with either a 0.1 or $0.3 \mathrm{~mm}$ reconstruction increment, and a H50 moderately sharp or H90 ultra sharp kernel.

\section{Measurements}

The CT scans were manually segmented in Amira (FEI Visualization Sciences Group, Berlin), then voxels were assigned to categories of cortical bone, enamel, marrow space, and dentin. All scans were transformed to Hounsfield units by assigning air and enamel values of -1000 and 6330 respectively, then linearly interpolating between these values. The value of 6330 was the average of all the enamel voxels in the mandibles scanned with a water phantom. This yielded values for cortical bone from 2800-3000. The air-filled marrow spaces in the dry mandibles were assigned the density of water (1 $\left.\mathrm{g} / \mathrm{cm}^{3}\right)$.

The relative locations of the following points were quantified using Amira: left and right supracondylar points (highest point on the mandibular condyles), infradentale, distal buccal cusp of right and left lower third molars $\left(M_{3}\right)$, mesial contact between crowns of right and left first incisors $\left(\mathrm{I}_{1}\right)$, and the most anterior 
point on the rim of mandibular foramen inferior to the lingula. Jaw length $\left(L_{j}\right.$ in $\mathrm{mm}$ ) was quantified as the distance from the midpoint between the right and left supracondylar points and infradentale. The occlusal plane was defined as the plane with vertices at the $M_{3}$ and $I_{1}$ points defined above (Fig. 2).

The location of the weighted center of mass $\left(\mathrm{CoM}_{w}\right)$ was calculated in Matlab (Mathworks $®$ Inc.) using information on the relative densities of the voxels. The mass of each voxel was estimated as its volume times its density and, because voxel volume is constant throughout the model

$$
C o M w=\text { mirimi }=(\rho i v) r i(\rho i v)=\rho i r i \rho i
$$

where $m_{i}, \rho_{i}$, and $r_{i}$, are the mass, density, and position vector of the $i$-th voxel of the model, respectively, and $v$ is the volume of each voxel. Assuming that Hounsfield units are linearly related to material density, we used Hounsfield units as a proxy for $\rho_{i}$.

The mass of each mandible $\left(M_{j}\right)$ in grams was measured using a scale sensitive to $0.01 \mathrm{~g}$.

\section{Moments of inertia}

We were interested in not only quantifying the scaling of the moment of inertia of the mandible, but also in isolating the determinants of that scaling. Hence, we separated the total moment of inertia of the mandible $\left(I_{j}\right)$ into $I_{x x}$, the rotational inertia of the mandible about a transverse axis (x-axis) passing through the weighted center of mass $\left(\mathrm{CoM}_{w}\right)$, and the rotational inertia of the mass of the mandible, concentrated at $C o M_{w}$, about the estimated axis of rotation $\left(l_{\text {axis }}\right)$.

The moment of inertia of a rigid body $(I)$, its resistance to angular acceleration about an axis of rotation, is defined as:

$I=$ miri $2=$ vi $\rho$ iri $2=$ vpiri 2

where $r$ is the distance of the $i$-th element to the axis of rotation. If $M_{j}$ is 
we can solve for $v$ as

$v=M j \rho i$

[6]

and substituting [6] into [4], the moment of inertia can be calculated as

$I=M \rho i r i 2 \rho i$.

[7]

The inertia tensor about the $\mathrm{CoM}_{w}(I)$ was calculated as described in the SOM Appendix, where

$I x x=M \rho i y i 2+z i 2 \rho i$

[8]

is the moment of inertia about an axis orthogonal to the mid-sagittal plane through $C o M_{w}$. Hence, $I_{x x}$ represents the rotational inertia of the mandible about an axis parallel to the largest rotation component during fast open and fast close phases of the gape cycle.

To estimate the rotational inertia of the mass of the mandible concentrated at $C o M_{w}$ about the actual axis of rotation $\left(I_{\text {axis }}\right)$, we needed to identify morphological features of the mandible that might serve as reasonable proxies for the location of the axis of rotation during the fast open and fast close phases of the gape cycle (Ross et al., 2012). These published and our unpublished data suggest that during the fast open and fast close phases of the gape cycle, the distance from $\mathrm{CoM}_{w}$ to the axis of rotation lies within the range defined by axes through four pairs of morphological points (Figs. 2 and 3).

1. $A_{s c}$, supracondylar axis, through the most superior points on the two mandibular condyles;

2. $A_{m f}$, mandibular foramen axis, through the anterior rims of the mandibular foramina; 
3. $A_{s c-o}$, horizontal axis, in the posteriorly projected occlusal plane, through the inferior projection of the midpoint of the supracondylar axis onto the occlusal plane;

4. $A_{m f-o}$, horizontal axis, in the posteriorly projected occlusal plane, through the inferior projection of the midpoint of the mandibular foramen axis onto the occlusal plane.

Using these four axes, four rotational inertia terms were calculated: i.e., $M_{j} L_{A s c}{ }^{2}$, $M_{j} L_{A m f}{ }^{2}, M_{j} L_{A s c-0}{ }^{2}$, and $M_{j} L_{\text {Amf-o }}$. Adding $I_{x x}$ to each of these yields four separate estimates of the rotational inertia of the mandible for each specimen:

1. $I_{A s c}=I_{x x}+\left(M_{j} L_{A s c}{ }^{2}\right)$

2. $I_{A m f}=I_{x x}+\left(M_{j} L_{A m f}{ }^{2}\right)$

3. $I_{\text {Asc-o }}=I_{x x}+\left(M_{j} L_{A s c-0}{ }^{2}\right)$

4. $I_{\text {Amf }-O}=I_{x x}+\left(M_{j} L_{A m f-O}{ }^{2}\right)$

\section{Statistical analyses}

To address the potential issue of phylogenetic non-independence of the data, all scaling analyses were performed using phylogenetic generalized least squares (PGLS; Purvis and Rambaut, 1995; Orme et al., 2012). PGLS regressions were generated using species means and regression models were fit using the CAPER package in $\mathrm{R}$ (Ver. 3.3.1). The data were modeled using PGLS because: our assignment of independent and dependent variables is not arbitrary (i.e., our biological questions indicate that natural variation should be partitioned asymmetrically; Smith, 2009); and our independent variable-jaw length-is a single measurement easy to make with high precision, whereas our dependent variables are estimated using multi-step processes involving CT scanning and image processing that we assume introduce more error. ${ }^{2}$ Because phylogenetic relationships and branch lengths are never known with certainty and results may vary depending on the phylogenetic inference that is used (Huelsenbeck et al., 2000; Lutzoni et al., 2001; Pagel and Lutzoni, 2002), we

\footnotetext{
${ }^{2} \mathrm{We}$ also modeled the data using phylogenetic reduced major axis regression (phyl.RMA) using the phytools package in $R$ (Revell, 2012). The two phylogenetic regression approaches yielded similar results and we report the phyl.RMA results in SOM.
} 
used a random sample of 100 dated phylogenies from a recent Bayesian inference of primate phylogeny (Arnold et al., 2010), using Version 3 of 10kTrees (http://10ktrees.fas. harvard.edu/). We used a maximum likelihood approach to estimate $\lambda$, a measure of phylogenetic signal, and log likelihood ratio tests to determine whether $\lambda$ differs significantly from 0 or 1 (Freckleton et al., 2002). If there is no phylogenetic correlation, then $\lambda=0 . \lambda>0$ indicates increasing phylogenetic dependence, with $\lambda=1$ indicating that the given branch lengths adequately account for variation in the trait under a Brownian motion model of evolution. When the $p$-values from log likelihood ratio tests indicate that $\lambda$ is significantly $>0$ and $=1$, there is clear and significant phylogenetic nonindependence of the data. When $p$-values indicate $\lambda<1$ and $=0$, data can be unambiguously interpreted as phylogenetically independent. When these relationships are ambiguous (e.g., $\lambda$ does not differ significantly from either 0 or 1 ), the presence (or lack thereof) and magnitude of phylogenetic nonindependence is inconclusive.

To assess the degree to which the calculation of accurate moments of inertia improves the Spring Model of jaw oscillation, we expressed the scaling exponents of the terms in the model relative to $L_{j}$. We then calculated the expected scaling of $T_{c}$ to $L_{j}$ and this value was compared with our published data on scaling of primate chewing frequency: i.e., $T_{c}$ to $L_{j}^{0.58}$ (Ross et al., 2009b). Hypothesis 1 predicts that accurate measures of mandibular moment of inertia will decrease the Spring Model scaling exponent for primates from the values of 0.79 or 0.82 in Table 4 of Ross et al. (2009b) to the observed values of 0.5-0.6. To address this hypothesis, we used $t$-tests to compare PGLS regression coefficients to the expected slope for isometry. Hypothesis 2 predicts that the intercepts of the scaling equations for mandibular moment of inertia of anthropoids will be higher than those for prosimians. To address this hypothesis, we tested for significant differences in intercepts between anthropoids and prosimians using analysis of covariance on the phylogeny-adjusted data. Tests for homogeneity of slopes were performed first and if there were significant differences in slopes, we did not test for differences in intercepts. Hypothesis 3 
predicts that more accurate measures of mandibular moment of inertia will not improve the performance of the Spring Model, i.e., that the observed values will not fall within the range predicted by the model.

\section{Results}

All bivariate correlations are high and significant, the lowest being those for $L_{A m f}$ and $L_{A m f-o}$ for prosimians (0.764 and 0.824, respectively; Table 3). Maximum likelihood estimates of $\lambda$ range between 0 and 1 (Table 3 ). Across our primate sample as a whole, phylogenetic non-independence of our data ( $\lambda$ significantly higher than 0 ) is seen in 11 out of 14 cases $(79 \%)$. Across Anthropoidea, phylogenetic non-independence is uncommon (4/14 $=20 \%)$. There are no cases where $\lambda>0$ for our prosimian sample. Collectively, these results suggest that the use of PGLS is appropriate. We do, however, observe a relatively high proportion of equivocal cases, where $\lambda$ is not significantly different from either 0 or 1 ( $57 \%$ for anthropoids and $43 \%$ for prosimians). In these cases, phylogenetic correlation can neither be supported nor rejected. Lack of statistical power associated with a relatively small number of prosimian species likely accounts, in part, for these findings (Freckleton et al., 2002). In addition, there may be clade-specific patterns of phylogenetic correlation for the relationships evaluated in this study, which can only be resolved with the inclusion of additional taxa. (Following the suggestion of previous investigators [e.g., Price, 1997; Garland et al., 1999], we also present ordinary least squares results in the SOM: they do not materially alter the results of our hypothesis-testing.)

Jaw dry mass $\left(M_{j}\right)$ increases with positive allometry against jaw length $\left(L_{j}\right)$ in all three groups, significantly so across primates as a whole. However, the slopes for $M_{j}$ relative to $L_{i}$ are not significantly different from isometry in anthropoids and prosimians (Table 3, Fig. 5C). The positive allometry across primates as a whole is due to the fact that the slopes in both prosimians and anthropoids exceed isometry.

In all species, the $\mathrm{CoM}_{w}$ is located at the mesiodistal level of $\mathrm{M}_{2-3}$, inferior to the occlusal plane, on or close to the midsagittal plane (Fig. 4). Within 
anthropoids and prosimians, the distances from $\mathrm{CoM}_{w}$ to all four axes of rotation are isometric relative to jaw length $\left(L_{j}\right.$; Table 3 ; Fig. $\left.5 \mathrm{~A}, \mathrm{~B}\right)$. We note that the slopes for prosimians for $L_{A m f}$ and $L_{A m f-0}$ tend towards negative allometry, but the low correlations and wide 95\% confidence intervals (Cls) mean that we can rule out neither isometry nor positive allometry. Across Primates as a whole, $L_{A m f}$, $L_{\text {Asc-o }}$, and $L_{\text {Amf-o }}$ are isometric with $L_{j}$, while $L_{A s c}$ scales with significant positive allometry $(b=1.060)$. The widespread presence of condylar translation during chewing in primates suggests that $A_{s c}$ is not the location of the rotation axis in primates, and therefore we argue that, taken together, these results suggest isometry of the distance from the $\mathrm{CoM}_{w}$ to plausible axes of rotation.

In contrast to the slight positive allometry or isometry of $M_{j}$ and the isometry of the distance from the $C o M_{w}$ to the axes of rotation, $I_{x x}$, the rotational inertia of the mandible about a transverse axis through $\mathrm{CoM}_{w}$ scales with significant positive allometry in primates and anthropoids (Table 3; Fig. 6A) and the slope of $I_{x x}$ scaling exceeds $5.0(b=5.295)$ in prosimians (Table 3$)$. Similarly, the rotational inertias of the $C o M_{w}$ about the axes of rotation $-M_{j} L_{A s c}{ }^{2}, M_{j} L_{A s c-o}{ }^{2}$, $M_{j} L_{A m f}{ }^{2}, M_{j} L_{A m f-o}{ }^{2}$-are also positively allometric (Fig. $6 \mathrm{~B}, \mathrm{C}$ ). All slope values are greater than 5.0 (Table 3), the primate slopes are all significantly positively allometric, and three of the anthropoid slopes are significantly positively allometric.

Because the total moment of inertia of the mandible is equal to $I_{x x}$ plus the moment of inertia of $M_{j} L_{A s c}{ }^{2}, M_{j} L_{A s c-0}{ }^{2}, M_{j} L_{A m f}{ }^{2}$, or $M_{j} L_{\text {Amf-o }}{ }^{2}$, and all of these parameters scale with positive allometry across primates, the overall moments of inertia of the mandible- $I_{A s c}, I_{A s c-0}, I_{A m f}, I_{A m f-o}$-are all positively allometric across primates (Fig. 7, Table 3). Across anthropoids, $I_{\text {Asc }}, I_{\text {Amf }}$, and $I_{\text {Amf }-O}$ are significantly positively allometric, but $I_{\text {Asc }}$ cannot be distinguished from isometry. The prosimian slopes for the inertia terms have wide confidence intervals and thus cannot be distinguished statistically from isometry or the anthropoid slopes (Table 3). Anthropoids and prosimians display significant differences in intercepts of the regression of both $I_{A m f}$ and $I_{A m f-O}$ against jaw length, with anthropoids having higher intercepts and larger moments of inertia than prosimians. 
Do more accurate moments of inertia improve the Spring Model's predictions of chew period scaling?

To determine whether more accurate estimates of $l_{j}$ improve the performance of the Spring Model, all the terms were expressed relative to $L_{j}$, and a new scaling exponent was calculated (Equation [9]):

$$
T_{c}=2 \pi \sqrt{\frac{M_{j} L_{j}^{2}}{k_{e} b_{e}{ }^{2}}} \rightarrow 2 \pi \sqrt{\frac{I_{j}}{\frac{A_{e}}{L_{m}} b_{e}{ }^{2}}} \rightarrow 2 \pi \sqrt{\frac{L_{j}^{5.3}}{\frac{L_{j}^{2.5}}{L_{j}^{1.0}}\left(L_{j}^{1.0}\right)^{2}}} \rightarrow T_{c} \alpha L_{j}^{0.9}
$$

In order for the scaling exponents predicted by the Spring Model to be improved by more accurate values of $l_{j}, l_{j}$ would have to scale with negative allometry relative to $L_{j}$ (i.e., with an exponent around 4.6-4.7 in order to yield $T_{c} \alpha L_{j}^{0.5-0.6}$ ). In fact, our estimates reveal positive allometry of $l_{j}$ relative to mandible length. The implications of this scaling relationship for the validity of the Spring Model can be assessed by using an exponent for $I_{j}$ of 5.3 , similar to that for $I_{\text {Amf }}$ for primates and anthropoids (Table 3), in Equation [9], one of many possible iterations of exponents from Table 4 of Ross et al. (2009b). Assuming positive allometry of jaw elevator muscle PCSA (exponent of 2.5), and isometry of muscle lengths and lever arms, yields $T_{c} \alpha L_{j}^{0.9}$, an exponent higher than any of those calculated by Ross et al. (2009b). More recent studies suggest that although clade-specific scaling relationships for jaw-muscle PCSAs include both positive and negative allometry ( Taylor et al., 2012, 2015; Taylor and Vinyard, 2013), the scaling exponent for jaw-muscle PCSAs across anthropoids is likely to be closer to isometry, i.e., $b=2.0$ (Taylor et al., 2013). Using an exponent of 2 for the scaling of jaw-muscle PCSA yields $T_{c} \alpha L_{j}^{1.5}$, an even worse fit of the Spring Model to the data. These results suggest that accurate estimates of rotational inertia of the mandible do not improve the Spring Model's predictions of the scaling of chew cycle duration in primates.

\section{Discussion}


Our first hypothesis was that the Spring Model would better predict the scaling of primate chew cycle period if the rotational inertia of the mandible was more accurately estimated. This would require that rotational inertia of primate mandibles scales with negative allometry. In fact, our results reveal that the overall rotational inertia of the primate mandible-estimated as $I_{A s c}, I_{A s c-o,} I_{A m f}$, and $I_{A m f-O}-$ actually scales with positive allometry relative to jaw length: i.e., as mandible length increases it becomes more difficult than predicted by isometry for larger primates to rotate their mandibles about horizontal axes. The positive allometry of the primate slopes suggests that, despite the small sample sizes for prosimians, this result is consistent across both suborders. Across primates as a whole, all inertia terms scale with statistically significant positive allometry. Moreover, across anthropoids, the moments of inertia about the two most realistic axes of rotation- $I_{\text {Amf }}, I_{\text {Amf-o }}$-are positively allometric. Across anthropoids, isometry is only found when the rotation axis is assumed to pass through the occlusal plane projection of the supracondylar axis, but in any event, the lower confidence limits of this slope only just include 5 . Thus, we argue that the data most strongly support the hypothesis that the rotational inertia of the primate mandible scales with positive allometry across primates, anthropoids, and probably prosimians. This does not corroborate the predictions of the first hypothesis.

Our second hypothesis was that the greater robusticity of anthropoid mandibles compared with prosimian mandibles is associated with higher moments of inertia in anthropoids. The small sample sizes and broad credibility intervals for the prosimian PGLS regression coefficients limit our ability to draw precise conclusions about the scaling of rotational inertia of prosimian mandibles. Nevertheless, slopes for our prosimian sample are similar to those calculated for anthropoids and primates, and display significantly lower intercepts for two of the estimates of moments of inertia $\left(I_{\text {Amf }}, I_{\text {Amf-o }} ;\right.$ Table 3$)$. Pending increases in prosimian sample sizes, we argue that our current results are at least consistent with the hypothesis that anthropoid mandibles have higher overall moments of inertia than prosimian mandibles of similar length (Table 3). In absolute terms, 
this difference ranges from $2.0-5.0 \times 10^{-6} \mathrm{~kg} \cdot \mathrm{m}^{2}$, equivalent to a difference in inertia produced by $2-5$ grams of material located $3 \mathrm{~cm}$ from the axis of rotation. In relative terms, the moment of inertia of the prosimian mandible is, on average, $70-75 \%$ that of the anthropoid mandible. The increased robusticity of the anthropoid mandible associated with deeper mandibular corpora and symphyses (Ravosa, 1991) certainly comes at the expense of an increased moment of inertia, but the biological significance of this expense remains to be demonstrated.

Why is rotational inertia of the mandible positively allometric?

Positive allometry of primate mandible rotational inertia is not due to allometry of the distance from the $\mathrm{CoM}_{w}$ to the axes of rotation because this distance is only positively allometric when the (inappropriate) supra-condylar axis is assumed to be the axis of rotation, and only across Primates as a whole, not within suborders. Positive allometry of mandibular rotational inertia is only partly due to positive allometry of mandibular mass relative to jaw length, because $M_{j}$ is only slightly positively allometric to $L_{j}$ across primates (Table 3 ). However, when jaw mass and distance to the rotational axes are combined into the $M_{j} L^{2}$ terms, positive allometry is the predominant signal, suggesting that the positive allometry of the overall inertia term $\left(l_{j}\right)$ is attributable at least in part to this slight positive allometry of jaw mass.

The most important contributor to the positive allometry of the overall inertia terms is $I_{x x}$, the rotational inertia of the mandible about a transverse axis through $\mathrm{CoM}_{\mathrm{w}}$. Compared with smaller primates, larger primates have their mandibular mass distributed about the center of mass in a manner that makes it more difficult for them to rotate their mandibles about a transverse axis through $C o M_{w}$. The combination of positive allometry of $I_{x x}$ and positive allometry of $M_{j}$ results in positive allometry of $I_{A s c}, I_{A s c-o}, I_{A m f}$, and $I_{A m f-O}$ across primates as a whole and of of $I_{A s c}, I_{A m f}$, and $I_{A m f-o}$ across anthropoids. The positive allometry of symphyseal dimensions within both anthropoids and prosimians (Ravosa, 1996a, b; Vinyard and Ravosa, 1998) cannot account for all of the positive allometry of 
moments of inertia because the distances from $\mathrm{COM}_{w}$ to the rotation axes are not positively allometric, as should be the case if size-related increases in symphyseal robusticity "pulled" the $\mathrm{COM}_{w}$ forward. Rather, the scaling of rotational inertia of primate mandibles is due to overall size-correlated changes in mandible shape, such as the positive allometry of ramus height seen across anthropoids (Ravosa et al., 2000). The impacts of variation in mandibular mass distribution on chewing mechanics in primates is discussed in the following section.

The relative unimportance of jaw momentum in chewing dynamics

Hypothesis 3 posited that mass-related momentum effects are of negligible importance in the scaling of primate chew cycle period. This hypothesis, based on the argument that the highly pinnate jaw-elevator muscles of primates afford them high passive stiffness (Ross et al., 2009b), is corroborated by our finding that incorporating more accurate estimates of moments of inertia in the Spring Model results in less accurate scaling of primate chew cycle period.

Comparison of the scaling of chew cycle period with locomotion step cycle period also suggests that mass-related momentum effects are of negligible importance in the scaling of primate chew period. Turvey et al. (1988) hypothesized that in locomotor systems: "as body mass decreases, the restoring moment relative to gravity becomes negligible in comparison with the restoring moment relative to the elasticity of the body's tissues" (311; see also Hooper, 2012). If increases in length of the limb are associated with increasing contributions of angular momentum to limb oscillation, but increases in jaw length are not similarly associated with increases in angular momentum effects in the chewing system, then limb cycle period should increase more slowly relative to limb length than does jaw cycle period relative to jaw length. This is exactly what the data indicate. The available step cycle period $\left(T_{s}\right)$ data reveal that $T_{s} \alpha L_{L}{ }^{0.32}$ during trotting and $\alpha L_{L}{ }^{0.38}$ during galloping; these exponents are not only lower than the $T_{s} \alpha L_{L}{ }^{0.5}$ predicted by pendulum mechanics (Hooper, 2012), but also 
lower than those reported for the scaling of chew cycle period (Fig. 8). Indeed, the distributions of limb step cycle periods and jaw chew cycle periods only overlap at the smallest limb lengths, where limb mechanics are argued to be dominated by muscle mechanics (Turvey et al., 1988; Hooper, 2012). These results are congruent with the hypothesis that angular momentum becomes increasingly important in driving limb oscillations with increases in limb length, but is less important in driving jaw oscillations.

Why do feeding systems not utilize momentum effects of pendulum mechanics for energetic savings? Locomotion consumes a large proportion of mammal energy budgets, so it is not surprising that walking and running stride frequencies are tuned to the natural frequencies of the body (Cavagna et al., 1997; Hunter and Smith, 2007; de Ruiter et al., 2013) and limbs (Marsh et al., 2004; Raichlen, 2004; Modica and Kram, 2005; Pontzer, 2007; Kilbourne and Hoffman, 2013a). In contrast, chewing utilizes a much smaller proportion of the overall energy budget than locomotion, so the importance of mandible oscillation energetics for feeding system design is less obvious (Ross and Iriarte-Diaz, 2014). One way to estimate the magnitude of this effect is to compare chewing energetics in anthropoids with those in prosimians: the greater rotational inertia of anthropoid mandibles predicts higher per cycle chewing costs for anthropoids. We expect that the absolute magnitude of this effect is small, especially compared with the costs of locomotion. In locomotion systems, use of angular momentum may also facilitate increases in limb oscillation speed, an important design criterion for escaping predators or agonistic conspecifics. In contrast, chewing speed is not an important design criterion in feeding systems of primates, because their feeding time is not tightly constrained (Ross et al., 2009a) and because we suspect that chewing too fast may expose the teeth to increased risk of breakage and wear.

Hence, we suggest that, whereas energetic efficiency and speed are important design criteria in mammal locomotion systems, control of tooth displacement and bite force production are more important in mammalian chewing. We hypothesize that the longer oscillation periods of mammal chewing 
function to protect the brittle teeth of adult mammals, which cannot be replaced, thereby minimizing fitness costs associated with excess tooth wear and breakage (Kojola et al., 1998; King et al., 2005). Mammal chew cycle periods relative to jaw length increase faster than step cycle periods relative to limb length, because it is less important for feeding systems to utilize angular momentum to conserve energy and more important to ameliorate tradeoffs between motor responsiveness and sensory resolution associated with increases in system size (More et al., 2010). In order to coordinate jaw, lip, cheek, and tongue movements during chewing, sensorimotor information must be integrated across at least three cranial nerve systems with nuclei located from the upper spinal cord (Yamada et al., 2005) to the cerebral cortex (Arce et al., 2013; Quintero et al., 2013). Loss of sensory resolution might impair the control of masticatory bite force and jaw kinematics (Lund, 1991; Trulsson, 2006), resulting in excessive feedback delays and increasing rates of self-injury during feeding in larger mammals. Thus, the need for better displacement and force control might explain not only the longer cycle periods of chewing compared with locomotion (Fig. 8), but the scaling of cycle periods within mammals as well.

Implications for primate and human evolution

The biomechanical, physiological, and ecological factors driving the evolution of musculoskeletal system design (form:function relationships) in primates are important foci of primate and human evolutionary studies, but it is not always obvious what the important optimality criteria are for any given system. Are the design principles in the locomotion system the same as those for the feeding system? What does variation in mandible morphology tell us about selective forces acting on primate feeding systems? Previous work has suggested that primate feeding time is not constrained in the wild, so that chewing speed is unlikely to be an important design criterion of the primate feeding system (Ross et al., 2009a). The results of this study suggest that gravity driven pendular mechanics are not important determinants of primate feeding system dynamics, even in the largest primates, implying that energetic efficiency 
may also be relatively less important than in the locomotion system. Instead, we hypothesize that minimization of the probability of tooth wear and breakage is a more important design criterion for primate feeding systems. To critically test these hypotheses, future work should assess the importance of energetics in primate feeding system function and investigate active and passive mechanisms for resisting tooth wear and breakage in primates.

\section{Conclusions}

The rotational inertia of primate mandibles scales with positive allometry across primates, anthropoids, and probably prosimians. This is due to the positive allometry of the moment of inertia of the mandible about a transverse axis through its center of mass, as well as positive allometry of mandibular mass relative to jaw length. Compared with prosimian mandibles of similar lengths, anthropoid mandibles exhibit greater rotational inertia around two rotational axes due to suborder differences in mandibular mass and the moment of inertia of the mandible about a transverse axis through its center of mass. The poor performance of the Spring Model, despite more accurate moment of inertia data, suggests that the determinants of primate chew period scaling lie with the scaling of aspects of muscle architecture and activity patterns that alter muscle stiffness. Differences in scaling of cycle periods between feeding and locomotor systems argue for the operation of different optimality criteria in the two systems. While locomotor systems are designed in part to minimize energy consumption and maximize speed, these criteria are less important in mammal feeding systems. Future work on differences in muscle architecture and control between feeding and locomotion systems promise to provide valuable insight into the hypotheses presented here.

\section{Acknowledgments}

We dedicate this paper to the memory of Bill Stanley, whose curation of the mammal collections at the FMNH has benefited our field enormously. L. Heaney and W. Stanley kindly facilitated the loan of specimens from the FMNH; 
C. Wietholt at University of Chicago scanned the FMNH specimens and transformed the image files into usable formats. The Smithsonian's Division of Mammals (Dr. Kristofer Helgen) and Human Origins Program (Dr. Matt Tocheri) provided scans of SMNH specimens (http://humanorigins.si.edu/evidence/3dcollection/primate) acquired through the generous support of the Smithsonian 2.0 Fund and the Smithsonian's Collections Care and Preservation Fund. Scott Hooper kindly provided data used in his 2012 paper for use in Figure 7. Nick Gidmark provided feedback on the manuscript. This research was supported by NSF award (including REU) 0962682 to CFR, AT, and Jonathan Perry.

\section{Appendix}

The inertia tensor about the $\mathrm{CoM}_{w}(I)$ was calculated as

$I=I x x I x y I x z I y x I y y I y z I z x I z y I z z$

where

$I x x=M \rho i y i 2+z i 2 \rho i$

$I y y=M \rho i x i 2+z i 2 \rho i$

$I z z=M \rho i x i 2+y i 2 \rho i$

$I x y=I y x=-M \rho i x i y i \rho i$

$I y z=I z y=-M \rho i y i z i \rho i$

$I x z=I z x=-M \rho i x i z i \rho i$,

the diagonal elements, $I_{x x}, I_{y y}$, and $I_{z z}$, are the moments of inertia about the $x-, y-$, and z-axes respectively, with the origin at $\mathrm{CoM}_{w}$, and the non-diagonal elements of the matrix are the products of inertia. The coordinate system of each mandible was defined such that $I_{x x}$ was about an axis orthogonal to the mid-sagittal plane. Hence, $I_{x x}$ represents the rotational inertia of the mandible about an axis through $\mathrm{CoM}_{w}$ and parallel to the largest rotation component during fast open and fast close phases of the gape cycle. 


\section{References}

Alexander, R.M., 2003. Principles of animal locomotion. Princeton University Press, Princeton.

Anapol, F., Shahnoor, N., Ross, C.F., 2008. Scaling of reduced physiologic cross-sectional area in primate muscles of mastication. In: Vinyard, C., Wall, C.E., Ravosa, M.J. (Eds.), Primate Craniofacial Function and Biology. Springer, New York, pp. 201-216.

Arce, F.I., Lee, J.C., Ross, C.F., Sessle, B.J., Hatsopoulos, N.G., 2013. Directional information from neuronal ensembles in the primate orofacial sensorimotor cortex. J. Neurophysiol. 110, 1357-1369.

Arnold, C., Matthews, L.J., Nunn, C.L., 2010. The 10kTrees website: a new online resource for primate phylogeny. Evol. Anthropol. 19, 114-118.

Biewener, A.A., 2005. Biomechanical consequences of scaling. J. Exp. Biol. 208, 1665-1676.

Cavagna, G.A., Mantovani, M., Willems, P.A., Musch, G., 1997. The resonant step frequency in human running. Pflügers Archaeol. 434, 678-684.

de Ruiter, C.J., Verdijk, P.W.L., Werker, W., Zuidema, M.J., de Haan, A., 2013. Stride frequency in relation to oxygen consumption in experienced and novice runners. Eur. J. Sport Sci. 14, 251-258.

Druzinsky, R.E., 1993. The time allometry of mammalian chewing movements: chewing frequency scales with body mass in mammals. J. Theor. Biol. 160, 427-440.

Fortelius, M., 1985. Ungulate cheek teeth: developmental, functional, and evolutionary interrelationships. Acta Zool. Fennica 180, 1-76.

Freckleton, R.P., Harvey, P.H., Pagel, M., 2002. Phylogenetic analysis and comparative data: A test and review of evidence. Am. Nat. 160, 712-726.

Gerstner, G.E., Gerstein, J.B., 2008. Chewing rate allometry among mammals. J. Mammal. 89, 1020-1030.

Gintof, C., Konow, N., Ross, C.F., Sanford, C.P.J., 2010. Rhythmicity in teleost chewing: A comparison with amniotes. J. Exp. Biol. 213, 1868-1875. 
Heglund, N.C., Taylor, C.R., 1988. Speed, stride frequency and energy cost per stride: how do they change with body size and gait? J. Exp. Biol. 138, 301-318.

Hooper, S.L., 2012. Body size and the neural control of movement. Curr. Biol. 22, R318-R322.

Houk, J.C., Rymer, W.Z., 1981. Neural control of muscle length and tension. In: Brooks, V.B. (Ed.), Handbook of Physiology-The Nervous System II. American Physiological Society, Bethesda, pp. 257-323.

Huelsenbeck, J.P., Rannala, B., Masly, J.P., 2000. Accommodating phylogenetic uncertainty in evolutionary studies. Science 288, 2349-2350.

Hunter, I., Smith, G., 2007. Preferred and optimal stride frequency, stiffness and economy: changes with fatigue during a 1-h high-intensity run. Eur. J. Appl. Physiol. 100, 653-661.

Kilbourne, B.M., 2014. Scale effects and morphological diversification in hindlimb segment mass proportions in neognath birds. Front. Zool. 11, 37.

Kilbourne, B.M., Hoffman, L.C., 2013. Scale effects between body size and limb design in quadrupedal mammals. PLoS ONE 8, e78392.

Kilbourne, B.M., Hoffman, L.C., 2015. Energetic benefits and adaptations in mammalian limbs: Scale effects and selective pressures. Evolution 69, 1546-1559.

King, S.J., Arrigo-Nelson, S.J., Pochron, S.T., Semprebon, G.M., Godfrey, L.R., Wright, P.C., Jernvall, J., 2005. Dental senescence in a long-lived primate links infant survival to rainfall. Proc. Natl. Acad. Sci. USA 102, 1657916583.

Kojola, I., Helle, T., Huhta, E., Niva, A., 1998. Foraging conditions, tooth wear and herbivore body reserves: a study of female reindeer. Oecologia 117, 26-30.

Lee, C.R., Farley, C.T., 1998. Determinants of the center of mass trajectory in human walking and running. J. Exp. Biol. 201, 2935-2944.

Lund, J.P., 1991. Mastication and its control by the brain stem. Crit. Rev. Oral. Biol. Med. 2, 33-64. 
Lutzoni, F., Pagel, M., Reeb, V., 2001. Major fungal lineages are derived from lichen symbiotic ancestors. Nature 411, 937-940.

Marsh, R.L., Ellerby, D.J., Carr, J.A., Henry, H.T., Buchanan, C.I., 2004. Partitioning the energetics of walking and running: swinging the limbs is expensive. Science 303, 80-83.

McMahon, T.A., 1975. Using body size to understand the structural design of animals: quadrupedal locomotion. J. Appl. Physiol. 39, 619-627.

McNab, B.K., 2002. The Physiological Ecology of Vertebrates. A View from Energetics. Cornell University Press, New York.

Modica, J.R., Kram, R., 2005. Metabolic energy and muscular activity required for leg swinging in running. J. Appl. Physiol. 98, 2126-2131.

More, H.L., Hutchinson, J.R., Collins, D.F., Weber, D.J., Aung, S.K., Donelan, J.M., 2010. Scaling of sensorimotor control in terrestrial mammals. Proceedings. Biol. Sci./Roy. Soc. 277, 3563-3568.

Nagamine, Y., Satoh, Y., Shimizu, D., Iwasaki, S.-i., Terada, K., 2013. Relationship between masticatory rhythm, body mass and mandibular morphology in primates. Arch. Oral Biol. 58, 1084-1091.

Nichols, T.R., 2002. The contributions of muscles and reflexes to the regulation of joint and limb mechanics. Clin. Orthop. Relat. Res., 403 S43-S50.

Norberg, R.A.., Aldrin, B.S.W., 2010. Scaling for stress similarity and distortedshape similarity in bending and torsion under maximal muscle forces concurs with geometric similarity among different-sized animals. J. Exp. Biol. 213, 2873-2888.

Orme, D., Freckleton, R., Thomas, G., Petzoldt, T., Fritz, S., Isaac, N., Pearse, W., 2012. CAPER: Comparative analyses of phylogenetics and evolution in R. R package., version 3.3.1. http.CRAN.R-project.org/package-caper

Pagel, M., Lutzoni, F., 2002. Accounting for phylogenetic uncertainty in comparative studies of evolution and adaptation. In: Lässig, M., Valleriani, A. (Eds.), Biological evolution and statistical physics. Springer-Verlag, Berlin, pp. 148-161. 
Pennycuick, C.J., 1975. Running of Gnu (Connochaetes-Taurinus) and other animals. J Exp. Biol. 63, 775-799.

Pontzer, H., 2007. Effective limb length and the scaling of locomotor cost in terrestrial animals. J. Exp. Biol. 210, 1752-1761.

Purvis, A., Rambaut, A., 1995. Comparative analysis by independent contrasts (CAIC): an Apple Macintosh application for analysing comparative data. Comp. Appl. Biosci. 11, 247-251.

Quintero, A., Ichesco, E., Myers, C., Schutt, R., Gerstner, G.E., 2013. Brain activity and human unilateral chewing: an fMRI study. J. Dent. Res. 92, 136-142.

Raichlen, D.A., 2004. Convergence of forelimb and hindlimb Natural Pendular Period in baboons (Papio cynocephalus) and its implication for the evolution of primate quadrupedalism. J. Hum. Evol. 46, 719-738.

Ravosa, M.J., 1991. Structural allometry of the mandibular corpus and symphysis in prosimian primates. J. Hum. Evol. 20, 3-20.

Ravosa, M.J., 1996a. Jaw scaling and biomechanics in fossil taxa. J. Hum. Evol. 30, 159-160.

Ravosa, M.J., 1996b. Mandibular form and function in North American and European Adapidae and Omomyidae. J. Morphol. 229, 171-190.

Ravosa, M.J., Vinyard, C.J., Gagnon, M., Islam, S.A., 2000. Evolution of anthropoid jaw loading and kinematic patterns. Am. J. Phys. Anthropol. 112, 493-516.

Reilly, S.M., McElroy, E.J., Biknevicius, A.R., 2007. Posture, gait and the ecological relevance of locomotor costs and energy-saving mechanisms in tetrapods. Zoology 110, 271-289.

Revell, L.J., 2012. phytools: An R package for phylogenetic comparative biology (and other things). Meth. Ecol. Evol. 3, 217-223.

Ross, C.F., Iriarte-Diaz, J., 2014. What does feeding system morphology tell us about feeding? Evol. Anthropol.: Issues News Rev. 23, 105-120.

Ross, C.F., Washington, R.L., Eckhardt, A., Reed, D.A., Vogel, E.R., Dominy, N.J., Machanda, Z.P., 2009a. Ecological consequences of scaling of chew 
cycle duration and daily feeding time in Primates. J. Hum. Evol. 56, 570585.

Ross, C.F., Reed, D.A., Washington, R.L., Eckhardt, A., Anapol, F., Shahnoor, N., 2009b. Scaling of chew cycle duration in primates. Am. J. Phys. Anthropol. 138, 30-44.

Ross, C.F., Iriarte-Diaz, J., Nunn, C.L., 2012. Innovative approaches to the relationship between diet and mandibular morphology in primates. Intl. J. Primatol. 33, 632-660.

Shipley, L.A., Gross, J.E., Spalinger, D.E., Hobbs, N.T., Wunder, B.A., 1994. The scaling of intake rate in mammalian herbivores. Am. Nat. 143, 1055-1082.

Smith, R.J., 2009. Use and Misuse of the Reduced Major Axis for Line-Fitting. Am. J. Phys. Anthropol. 140, 476-486.

Taylor, A.B., Vinyard, C.J., 2013. The relationships among jaw-muscle fiber architecture, jaw morphology and feeding behavior in extant apes and modern humans. Am. J. Phys. Anthropol. 151, 120-134.

Taylor, A.B., Yuan, T., Ross, C.F., Vinyard, C.J., 2012. Taylor AB, Yuan T, Ross CF, Vinyard CJ, 2012. Scaling of jaw-muscle fiber architecture in platyrrhines: a preliminary assessment. Am. J. Phys. Anthropol. (Suppl.) 54, 283.

Taylor, A.B., Yuan, T., Ross, C.F., Vinyard, C.J., 2013. The scaling of jaw-muscle fiber architecture in anthropoid primates. Am. J. Phys. Anthropol. (Suppl.) 56, 269.

Taylor, A.B., Yuan, T., Ross, C.F., Vinyard, C.J., 2015. Jaw-muscle force and excursion scale with negative allometry in platyrrhine primates. Am. J. Phys. Anthropol.158: 242-256.

Trulsson, M., 2006. Sensory-motor function of human periodontal mechanoreceptors. J. Oral Rehab. 33, 262-273.

Turvey, M.T., Schmidt, R.C., Rosenblum, L.D., Kugler, P.N., 1988. On the time allometry of co-ordinated rhythmic movements. J. Theor. Biol. 130, 285325. 
Vinyard, C.J., Ravosa, M.J., 1998. Ontogeny, function, and scaling of the mandibular symphysis in papionin primates. J. Morphol. 235, 157-175.

Wang, J.S., Stohler, C.S., 1991. Predicting foodstuff from jaw dynamics during masticatory crushing in man. Arch. Oral Biol. 36, 239-244.

Yamada, Y., Yamamura, K., Inoue, M., 2005. Coordination of cranial motoneurons during mastication. Resp. Physiol. Neurobiol. 147, 177-189. 
Table 1. Variables, abbreviations, and dimensionality.

\begin{tabular}{|c|c|}
\hline Symbol & Description (Dimensionality) \\
\hline & $L=$ length; $M=$ mass; $T=$ time \\
\hline \multicolumn{2}{|l|}{ Temporal variables } \\
\hline$F_{c}$ & Chew frequency $=1 / \mathrm{T}_{\mathrm{c}}\left(\mathrm{T}^{-1}\right)$ \\
\hline$F_{p}$ & Natural frequency of pendulum $=1 / \mathrm{T}_{\mathrm{p}}\left(\mathrm{T}^{-1}\right)$ \\
\hline$T_{c}$ & Chew cycle duration $(\mathrm{T})$ \\
\hline$T_{p}$ & Natural period (cycle duration) of a pendulum (T) \\
\hline$g$ & Acceleration due to gravity $\left(\mathrm{LT}^{-2}\right)$ \\
\hline \multicolumn{2}{|l|}{ Location variables } \\
\hline$A_{s c}$ & Supracondylar axis, through the most superior point on each mandibular condyle \\
\hline$A_{m f}$ & Mandibular foramen axis, through the most anterior point on the rim of each mandibular foramen \\
\hline$A_{s c-o}$ & Axis orthogonal to mid-sagittal plane through projection of midpoint of $\mathrm{A}_{\mathrm{sc}}$ onto occlusal plane \\
\hline$A_{m f-o}$ & Axis orthogonal to mid-sagittal plane through projection of midpoint of $A_{m f}$ onto occlusal plane \\
\hline CoM & Center of mass \\
\hline $\mathrm{CoM}_{w}$ & Weighted center of mass \\
\hline \multicolumn{2}{|l|}{$\begin{array}{l}\text { Displacement and } \\
\text { distance variables }\end{array}$} \\
\hline$b_{d}$ & Lever arm of jaw depressor muscles (L) \\
\hline$b_{e}$ & Lever arm of jaw elevator muscles (L) \\
\hline$b_{\text {mass, }}$ temp, med.pt. & Lever arm of masseter, temporalis, or medial pterygoid muscles (L) \\
\hline$L_{A m f}$ & Distance from $C o M_{w}$ to $A_{m f}$ midpoint $(\mathrm{L})$ \\
\hline$L_{\text {Amf-o }}$ & Distance from $\mathrm{CoM}_{w}$ to projection of $A_{m f}$ midpoint onto occlusal plane (L) \\
\hline$L_{A s c}$ & Distance from $\mathrm{CoM}_{w}$ to $A_{s c}$ midpoint (L) \\
\hline$L_{A s c-O}$ & Distance from $C o M_{w}$ to projection of $A_{s c}$ midpoint onto occlusal plane (L) \\
\hline$L_{j}$ & Jaw length (L) \\
\hline$L_{m}$ & Muscle length (L) \\
\hline$L_{p}$ & Pendulum length = distance from axis of rotation to center of mass (L) \\
\hline$r_{i}$ & Position vector of each voxel (L) \\
\hline \multicolumn{2}{|l|}{ Area variables } \\
\hline$A_{e}$ & PCSA of jaw elevator muscles $\left(\mathrm{L}^{2}\right)$ \\
\hline$P C S A$ & Physiologic cross-sectional area $\left(\mathrm{L}^{2}\right)$ \\
\hline \multicolumn{2}{|l|}{$\begin{array}{l}\text { Mass, volume and } \\
\text { density variables }\end{array}$} \\
\hline $\mathrm{g}$ & Grams (M) (cf. italicized $g$, representing acceleration due to gravity) \\
\hline$M_{b}$ & Body mass (M) \\
\hline$m_{i}$ & Mass of individual voxel (M) \\
\hline$M_{j}$ & Jaw mass $(\mathrm{M})$ \\
\hline$M_{p}$ & Pendulum mass (M) \\
\hline
\end{tabular}




\begin{tabular}{|l|l|}
\hline$\rho_{i}$ & Density of individual voxel $\left(\mathrm{M} / \mathrm{L}^{3}=\mathrm{L}^{1}\right)$ \\
\hline$v$ & Volume of each voxel $\left(\mathrm{L}^{3}\right)$ \\
\hline Force variables & \\
\hline$k$ & Spring constant $\left(\mathrm{ML}^{-1} \mathrm{~T}^{-2}\right)$ \\
\hline$k_{L}, k_{e}$ & Spring constant of limb muscles, spring constant of jaw elevator muscles \\
\hline$F_{b}$ & Bite force $\left(\mathrm{MLT}^{-2}\right)$ \\
\hline$E$ & Slope of stress strain curve, i.e., Young's modulus for calculation of $k$ for muscles $\left(\mathrm{ML}^{-1} \mathrm{~T}^{-2}\right)$ \\
\hline Moments of inertia & $\left(\mathrm{ML}{ }^{2}=\mathrm{L}^{5}\right)$ \\
\hline$I_{A s c}$ & $I_{x x}+\left(M j L_{A s c}{ }^{2}\right)$, mandible's moment of inertia about $A_{s c}\left(\mathrm{ML}^{2}=\mathrm{L}^{5}\right)$ \\
\hline$I_{A s c-o}$ & $I_{x x}+\left(M j L_{A s c-o}{ }^{2}\right)$, mandible's moment of inertia about $A_{s c-0}\left(\mathrm{ML}^{2}=\mathrm{L}^{5}\right)$ \\
\hline$I_{A m f}$ & $I_{x x}+\left(M j L_{A m f}\right)$, mandible's moment of inertia about $A_{m f}\left(\mathrm{ML}^{2}=\mathrm{L}^{5}\right)$ \\
\hline$I_{A m f-o}$ & $I_{x x}+\left(M j L_{A m f-o}{ }^{2}\right)$, mandible's moment of inertia about $A_{m f-0}\left(\mathrm{ML}^{2}=\mathrm{L}^{5}\right)$ \\
\hline$I_{j}$ & Moment of inertia of the mandible $\left(\mathrm{ML}^{2}=\mathrm{L}^{5}\right)$ \\
\hline$I_{p}$ & Moment of inertia of a pendulum $\left(\mathrm{ML}^{2}=\mathrm{L}^{5}\right)$ \\
\hline$I_{x x}, I_{v v}, I_{z z}$ & Moments of inertia about x (mediolateral), y (superoinferior), z (rostrocaudal) axes $\left(\mathrm{ML}^{2}=\mathrm{L}^{5}\right)$ \\
\hline$O p e r a t o r$ & \\
\hline$\alpha$ & Proportional to \\
\hline
\end{tabular}


Table 2. Specimens and species sampled in this study. ${ }^{\text {a }}$

\section{Anthropoids}

Allenopithecus nigroviridis SMNH 395131

Aotus trivirgatus nigriceps FMNH 84226

Aotus trivirgatus nigriceps FMNH 90019

Ateles geoffroyi FMNH 13898

Cebuella pygmaea FMNH 87137

Chiropotes satanas FMNH 46179

Colobus guereza FMNH 35116

Gorilla gorilla SMNH 582726

Hylobates concolor FMNH 31768

Hylobates lar entelloides male SMNH 111989

Hylobates lar FMNH 99750

Hylobates lar FMNH 99751

Hylobates lar vestitus SMNH 143569

Hylobates lar vestitus SMNH 143570

Lagothrix cana FMNH 98056

Macaca fascicularis FMNH 87420

Macaca fascicularis philippinensis SMNH

256072

Macaca fascicularis philippinensis SMNH

573504

Mandrillus sphinx SMNH 283109

Mandrillus sphinx SMNH 598494

Miopithecus ogouensis SMNH 598519

Miopithecus ogouensis SMNH 598534

Miopithecus talapoin FMNH 83634

Nomascus concolor SMNH 320786

Nomascus concolor SMNH 320787

Pan troglodytes SMNH 395820

Pan troglodytes SMNH 599172 
Papio anubus SMNH 162899

Papio anubus SMNH 397476

Papio papio SMNH 381430

Pithecia monachus FMNH 122798

Pithecia monachus FMNH 25323

Pongo abelii SMNH 143590

Pongo abelii SMNH 143601

Pongo pygmaeus SMNH 142190

Pongo pygmaeus SMNH 142200

Saguinus oedipus geoffroyi SMNH 306845

Saimiri sciureus sciureus SMNH 338948

Symphalangus syndactylus FMNH 60340

Symphalangus syndactylus SMNH 114497

Symphalangus syndactylus SMNH 283563

Theropithecus gelada gelada SMNH 305107

Theropithecus gelada gelada SMNH 319992

\section{Prosimians}

Cheirogaleus medius FMNH 147986

Loris tardigradus FMNH 95028

Microcebus murinus FMNH 161647

Nycticebus pygmaeus FMNH 46826

Otolemur crassicaudatus FMNH 208695

Otolemur crassicaudatus FMNH 96274

Propithecus coquereli FMNH 8344

Propithecus verreauxi FMNH 8342

Tarsius syrichta FMNH 56159

${ }^{a}$ FMNH = Field Museum of Natural History, Chicago, IL; SMNH = Smithsonian Museum of Natural History, Washington, DC 
Table 3. Relationships of mass, distance, and moments of inertia variables to jaw length $\left(L_{j}\right)$ estimated using PGLS ${ }^{\text {a-d }}$

\begin{tabular}{|c|c|c|c|c|c|c|c|c|c|}
\hline & $\mathbf{R}^{2}$ & Slope & $\begin{array}{c}95 \% \text { CIs for the } \\
\text { slopes }\end{array}$ & Intercept & $\begin{array}{l}\text { 95\% CIs for } \\
\text { intercepts }\end{array}$ & $\lambda$ & $\begin{array}{c}\text { Lower bound } \lambda \\
p \text {-value }\end{array}$ & $\begin{array}{c}\text { Upper bound } \lambda \\
p \text {-value }\end{array}$ & $\begin{array}{c}P \text {-value } \\
\text { slope/intercept } \\
\text { differences of } \\
\text { anthropoids vs. } \\
\text { prosimians } \\
\end{array}$ \\
\hline \multicolumn{10}{|l|}{$M j$} \\
\hline Primates & 0.972 & 3.229 & $3.062-3.446$ & 1.962 & $1.705-2.292$ & 1.00 & 0.0114 & ns & \\
\hline Anthropoids & 0.971 & 3.183 & $2.970-3.450$ & 1.964 & $1.679-2.287$ & 1.00 & 0.0136 & $\mathrm{~ns}$ & $\mathrm{~ns} / \mathrm{ns}$ \\
\hline Distance terms & & Isometry $=1.0$ & & & & & & & \\
\hline \multicolumn{10}{|l|}{$L_{A s c}$} \\
\hline Primates & 0.989 & 1.060 & $1.018-1.102$ & -0.163 & $-0.225-0.097$ & 0.83 & 0.0001 & 0.0447 & \\
\hline Anthropoids & 0.987 & 1.045 & $0.993-1.101$ & -0.157 & $-0.222-0.089$ & 0.86 & 0.0055 & ns & $\mathrm{ns} / \mathrm{ns}$ \\
\hline \multicolumn{10}{|l|}{$L_{\text {Amf }}$} \\
\hline Prosimians & $0.764 *$ & 0.847 & $0.360-1.314$ & -0.797 & $-1.494-0.102$ & 0.00 & ns & 0.0589 & \\
\hline \multicolumn{10}{|l|}{$L_{A s c-o}$} \\
\hline Primates & 0.989 & 0.956 & $0.917-0.996$ & -0.339 & $-0.396-0.281$ & 0.23 & $\mathrm{~ns}$ & 0.0001 & \\
\hline Anthropoids & 0.991 & 0.918 & $0.880-0.983$ & -0.375 & $-0.423-0.290$ & 0.00 & ns & 0.0011 & $0.028 /---$ \\
\hline Prosimians & 0.998 & 1.031 & $0.982-1.086$ & -0.238 & $-0.309-0.154$ & 0.00 & ns & 0.0003 & \\
\hline \multicolumn{10}{|l|}{$L_{A m f-O}$} \\
\hline Primates & 0.929 & 1.014 & $0.921-1.124$ & -0.505 & $-0.643-0.348$ & 0.80 & $\mathrm{~ns}$ & 0.0407 & \\
\hline Anthropoids & 0.939 & 1.027 & $0.911-1.148$ & -0.466 & $-0.612-0.313$ & 0.83 & ns & $\mathrm{ns}$ & $\mathrm{ns} / \mathrm{ns}$ \\
\hline Prosimians & $0.824 *$ & 0.886 & $0.520-1.276$ & -0.715 & $-1.284-0.110$ & 0.05 & ns & 0.0414 & \\
\hline
\end{tabular}


Table 3 continued

\begin{tabular}{|c|c|c|c|c|c|c|c|c|c|}
\hline & $\mathbf{R}^{2}$ & Slope & $\begin{array}{c}\begin{array}{c}95 \% \text { CIs for } \\
\text { slopes }\end{array} \\
\end{array}$ & Intercept & $\begin{array}{c}\text { 95\% CIs for } \\
\text { intercepts }\end{array}$ & $\lambda$ & $\begin{array}{c}\text { Lower bound } \\
\lambda p \text {-value }\end{array}$ & $\begin{array}{c}\text { Upper } \\
\text { bound } \lambda p \text { - } \\
\text { value }\end{array}$ & $\begin{array}{c}p \text {-value slope/intercept } \\
\text { differences of anthropoids } \\
\text { vs. prosimians } \\
\end{array}$ \\
\hline Inertia terms & & Isometry $=5.0$ & & & & & & & \\
\hline \multicolumn{10}{|l|}{$I_{x x}$} \\
\hline Primates & 0.986 & 5.342 & $5.097-5.565$ & 1.002 & $0.627-1.328$ & 0.72 & 0.0283 & ns & \\
\hline Anthropoids & 0.986 & 5.302 & $5.031-5.576$ & 1.019 & $0.660-1.386$ & 0.70 & ns & ns & $\mathrm{ns} / \mathrm{ns}$ \\
\hline Prosimians & 0.980 & 5.295 & $4.554-6.164$ & 0.879 & $-0.231-2.209$ & 0.00 & $\mathrm{~ns}$ & ns & \\
\hline \multicolumn{10}{|l|}{$M_{j} L_{A s c}{ }^{2}$} \\
\hline Primates & 0.983 & 5.358 & $5.133-5.643$ & 1.650 & $1.274-2.066$ & 0.95 & 0.0015 & $\mathrm{~ns}$ & \\
\hline Anthropoids & 0.981 & 5.285 & $5.020-5.625$ & 1.669 & $1.283-2.083$ & 0.95 & 0.0125 & ns & $\mathrm{ns} / \mathrm{ns}$ \\
\hline Prosimians & 0.987 & 5.611 & 4.805-6.149 & 1.979 & $0.737-2.799$ & 1.00 & $\mathrm{~ns}$ & $\mathrm{~ns}$ & \\
\hline \multicolumn{10}{|l|}{$M_{j} L_{A m f^{2}}$} \\
\hline Primates & 0.985 & 5.334 & $5.074-5.587$ & 1.017 & $0.658-1.382$ & 0.76 & 0.0052 & 0.0313 & \\
\hline Anthropoids & 0.994 & 5.303 & $5.057-5.568$ & 1.072 & $0.767-1.416$ & 0.00 & ns & ns & $\mathrm{ns} / \mathrm{ns}$ \\
\hline Prosimians & 0.970 & 5.086 & $4.195-5.963$ & 0.585 & $-0.726-2.008$ & 0.64 & ns & 0.0996 & \\
\hline \multicolumn{10}{|l|}{$M_{i} L_{A s c-o}{ }^{2}$} \\
\hline Primates & 0.990 & 5.203 & $5.003-5.392$ & 1.363 & $1.057-1.638$ & 0.70 & 0.0111 & ns & \\
\hline Anthropoids & 0.990 & 5.110 & $4.865-5.352$ & 1.323 & $1.038-1.638$ & 0.65 & ns & ns & $\mathrm{ns} / \mathrm{ns}$ \\
\hline Prosimians & 0.990 & 5.391 & $4.945-5.949$ & 1.587 & $0.894-2.458$ & 0.00 & ns & ns & \\
\hline \multicolumn{10}{|l|}{$M_{j} L_{A m f-o}{ }^{2}$} \\
\hline Primates & 0.989 & 5.298 & $5.091-5.506$ & 1.009 & $0.684-1.313$ & 0.82 & 0.0007 & 0.0482 & \\
\hline Anthropoids & 0.996 & 5.241 & $5.075-5.493$ & 1.030 & $0.813-1.340$ & 0. & $\mathrm{~ns}$ & 0.0224 & $\mathrm{~ns} / \mathrm{ns}$ \\
\hline Prosimians & 0.975 & 5.163 & $4.337-6.000$ & 0.748 & $-0.524-2.036$ & 0.64 & ns & 0.0910 & \\
\hline \multicolumn{10}{|l|}{$I_{A s c}$} \\
\hline Primates & 0.984 & 5.354 & $5.135-5.617$ & 1.738 & $1.386-2.144$ & 0.93 & 0.0027 & $\mathrm{~ns}$ & \\
\hline Anthropoids & 0.982 & 5.287 & $4.987-5.597$ & 1.756 & $1.355-2.153$ & 0.94 & 0.0168 & ns & $\mathrm{ns} / \mathrm{ns}$ \\
\hline
\end{tabular}




\section{Table 3 continued}

\begin{tabular}{|c|c|c|c|c|c|c|c|c|c|}
\hline & $\mathbf{R}^{2}$ & Slope & 95\% CIs for slopes & Intercept & $\begin{array}{c}95 \% \text { CIs for } \\
\text { intercepts }\end{array}$ & $\lambda$ & $\begin{array}{c}\text { Lower bound } \\
\lambda p \text {-value }\end{array}$ & $\begin{array}{c}\text { Upper bound } \lambda \\
p \text {-value }\end{array}$ & $\begin{array}{c}p \text {-value } \\
\text { slope/intercept } \\
\text { differences of } \\
\text { anthropoids vs } \\
\text { prosimians } \\
\end{array}$ \\
\hline \multicolumn{10}{|l|}{$I_{\text {Amf }}$} \\
\hline Primates & 0.991 & 5.339 & $5.134-5.547$ & 1.320 & $1.058-1.628$ & 0.69 & 0.0061 & 0.0382 & \\
\hline Anthropoids & 0.992 & 5.320 & $5.082-5.542$ & 1.373 & $1.076-1.656$ & 0.42 & ns & ns & $\mathrm{ns} / 0.0018$ \\
\hline Prosimians & 0.987 & 5.132 & $4.477-5.853$ & 0.947 & $-0.028-2.058$ & 0.00 & ns & 0.0250 & \\
\hline \multicolumn{10}{|l|}{$I_{\text {Asc-o }}$} \\
\hline Primates & 0.990 & 5.230 & $5.023-5.417$ & 1.508 & $1.198-1.790$ & 0.76 & 0.0100 & ns & \\
\hline Anthropoids & 0.988 & 5.143 & $4.923-5.392$ & 1.477 & $1.189-1.785$ & 0.82 & ns & ns & $\mathrm{ns} / \mathrm{ns}$ \\
\hline Prosimians & 0.989 & 5.368 & 4.841-6.019 & 1.659 & $0.884-2.659$ & 0.00 & ns & ns & \\
\hline \multicolumn{10}{|l|}{$I_{A m f-O}$} \\
\hline Primates & 0.991 & 5.320 & $5.144-5.513$ & 1.315 & $1.056-1.590$ & 0.73 & 0.0047 & ns & \\
\hline Anthropoids & 0.996 & 5.295 & $5.077-5.492$ & 1.359 & $1.086-1.621$ & 0.47 & ns & ns & $\mathrm{ns} / 0.0016$ \\
\hline Prosimians & 0.986 & 5.172 & $4.543-5.856$ & 1.030 & $0.089-2.101$ & 0.00 & ns & 0.0309 & \\
\hline
\end{tabular}

${ }^{a}$ All correlations (R) significant at $p<0.0001$, except those marked with ${ }^{*}$, which are significant at $p<0.01$.

${ }^{\mathrm{b}}$ Boldfaced slopes depart significantly from isometry at $p<0.05$.

${ }^{\mathrm{c} B e c a u s e}$ of the small sample size for prosimians, we provide $p$-values for lambda when $0.05<p<1.0$.

${ }^{\mathrm{d}} 95 \%$ Cls estimated using the Markov Chain Monte Carlo method using the BayesTraits Wrapper package in $R$.

Iterations: 1010000; Burn in: 10000; Sample 1000. 

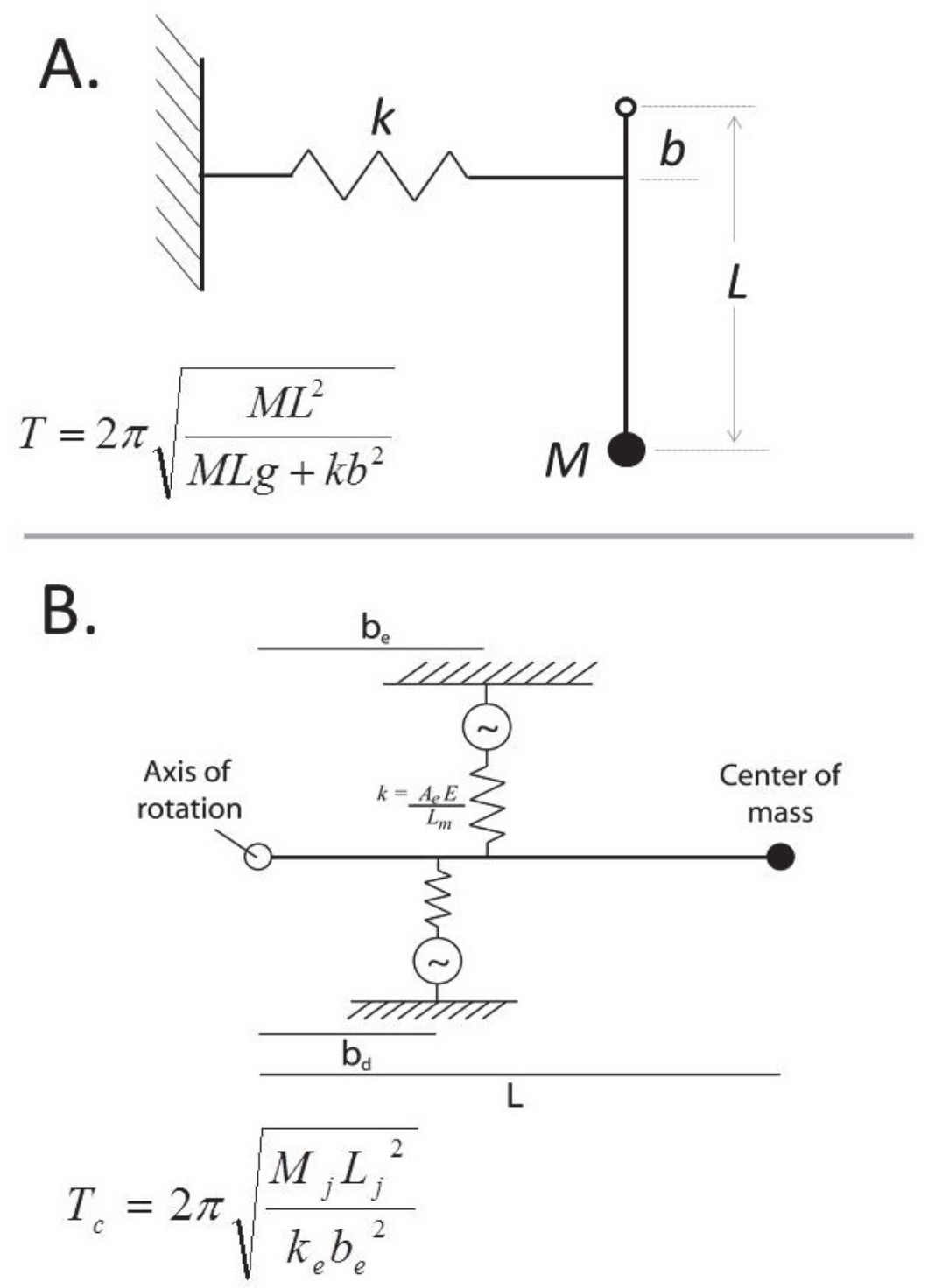

Figure 1. A. Turvey et al.'s (1988) hybrid mass-spring/simple pendulum model, together with the equation describing its natural period. The pendulum is a stiff, weightless rod of length $L_{p}$ carrying a mass, $M_{p}$; a spring attached at distance $b$ from the axis of rotation provides a restoring torque; gravity, $g$, acting on $M$ at length $L$ provides the driving torque.

B. Spring Model of the jaw system presented by Ross et al. (2009b), together with the equation describing its natural period. $L$ is jaw length, $b_{e}$ and $b_{d}$ are the jaw elevator and depressor lever arms respectively, and $k=E A_{e} / L_{m}$ is a spring constant representing the jaw-elevator muscles, where $E$ is muscle stiffness, $A_{e}$ is physiological cross-sectional area of the muscle, and $L_{m}$ is muscle length (McMahon, 1975, 1984). 


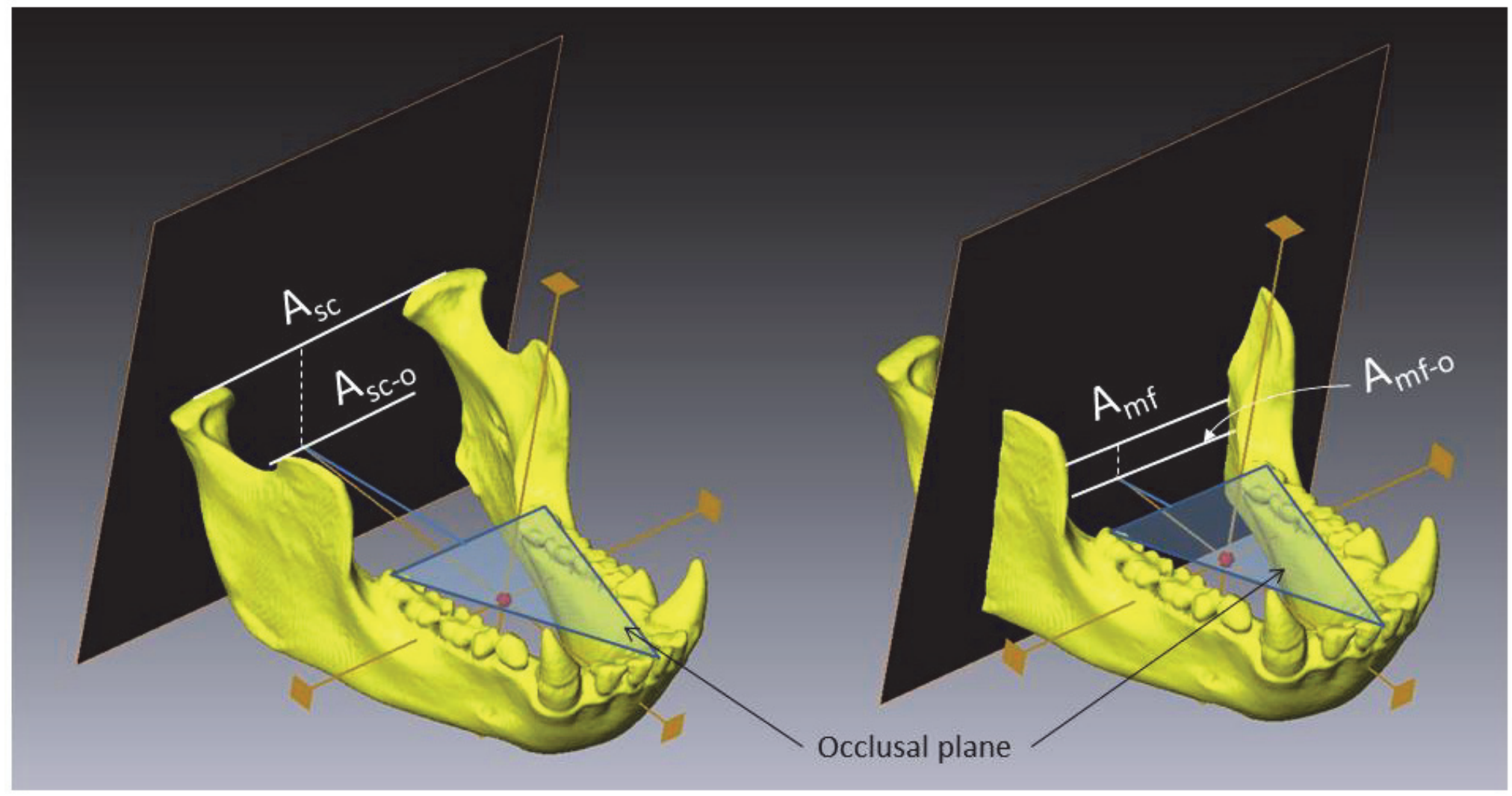

Figure 2. Axes and planes used in this study. $A_{s c}$, supracondylar axis, through the most superior point on the two mandibular condyles; $A_{m}$, mandibular foramen axis, through the anterior rims of the mandibular foramina; $A_{s c-o}$, axis through the intersection of the projection of the midpoint of the supracondylar axis with the posterior projection of the occlusal plane; $A_{m f-o}$, axis through the intersection in the inferior projection of the midpoint of the mandibular foramen axis with the posterior projection of the occlusal plane. 

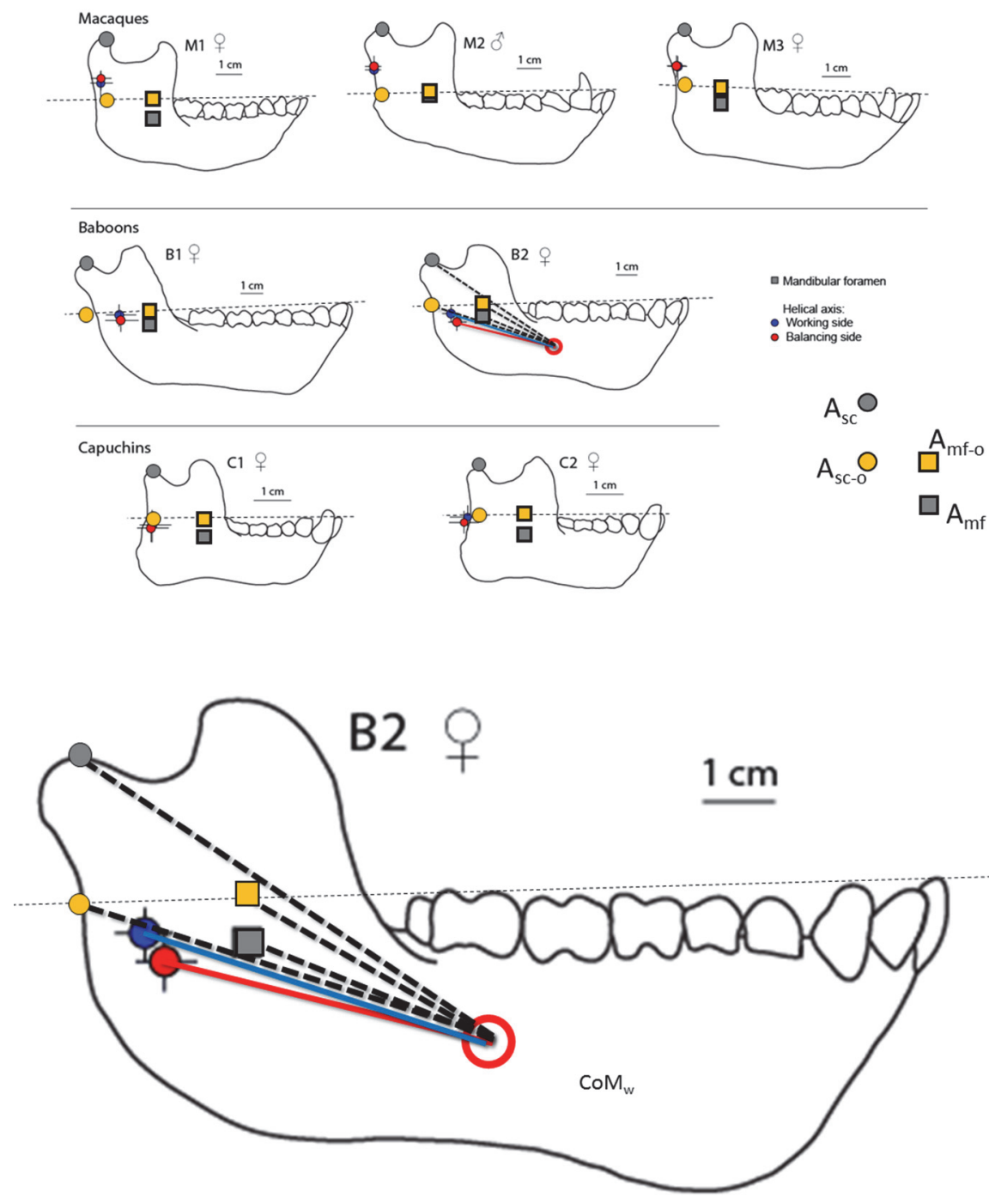

Figure 3. Average location of the intersection of the finite helical axis of the mandible with the ramus on the balancing side (red dot) and working side (blue dot) during fast close and fast open. The top panel shows averages for three macaques, two baboons, and two capuchins. The colored circles and squares indicate the location of the morphological features (gray) and their occlusal plane projections (yellow) used in this study to calculate the distance from the weighted center of mass to the axis of rotation. The bottom image shows a close-up of the mandible of baboon 2 (female). The dashed lines in the bottom image and the middle image in the top panel show the distances from the weighed center of mass $\left(\mathrm{CoM}_{w}\right.$; red target) to the midline projections of these four points, along with the distances to the midline projection of balancing and working side axes. The distances to the actual helical axis always lie within the bounds set by the distances to the four points. 


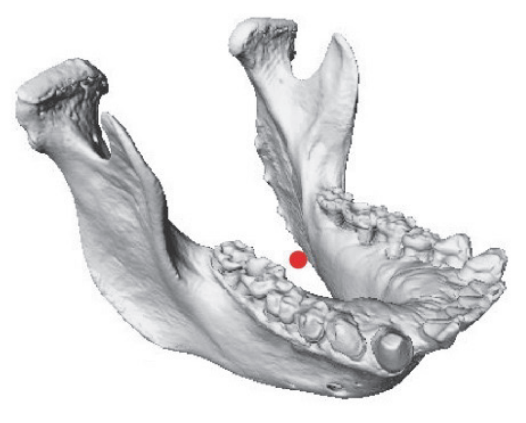

Gorilla gorilla

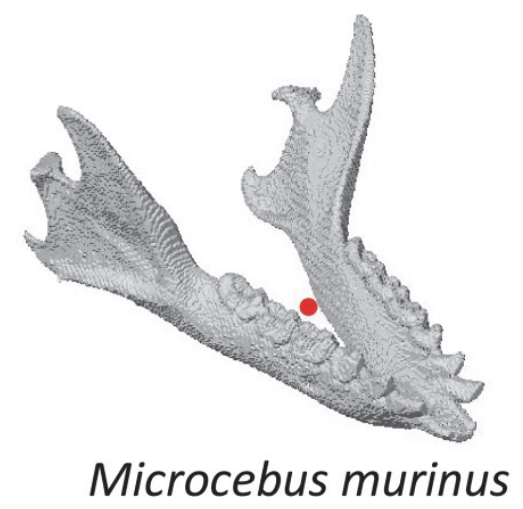

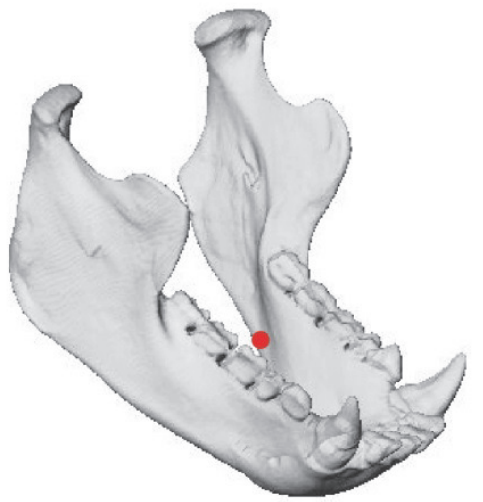

Pan troglodytes

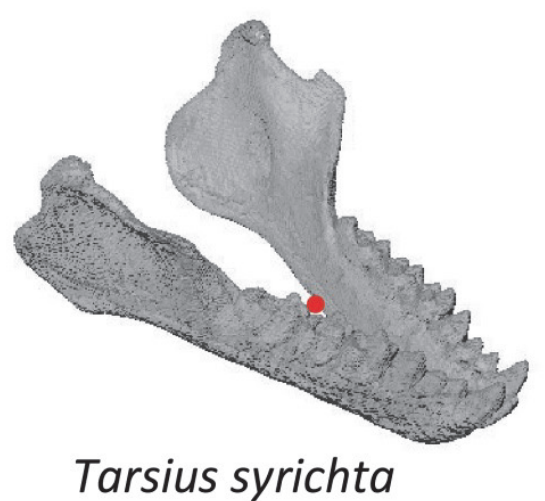

Figure 4. Location of weighted center of mass ( $\mathrm{CoM}_{w}$; red point) in four species of primates. 
A

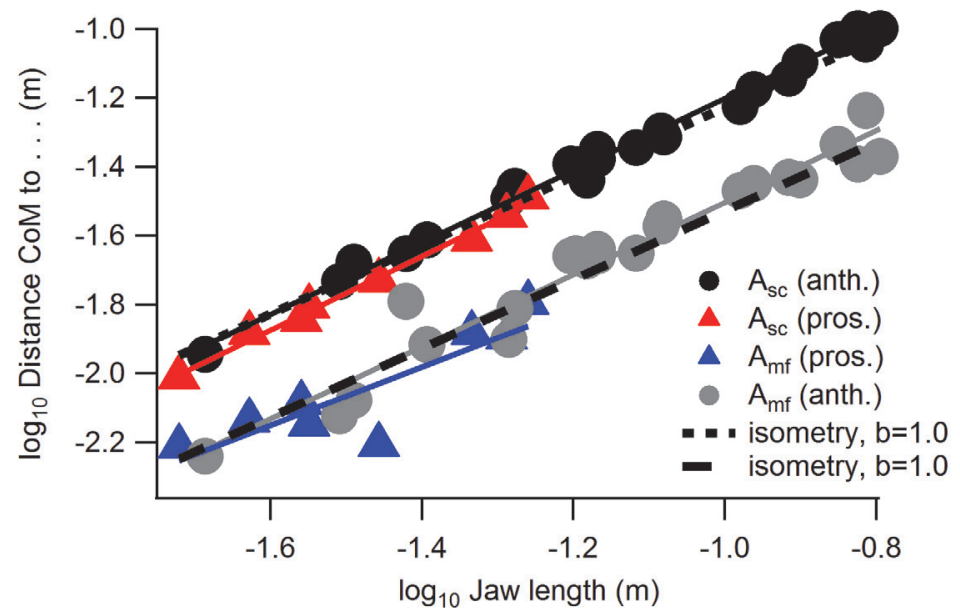

B

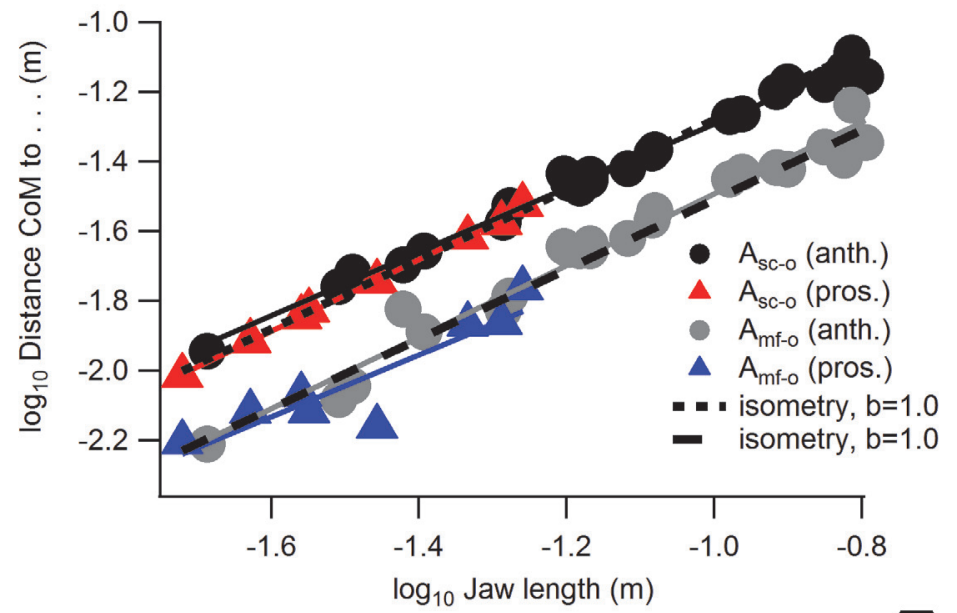

C

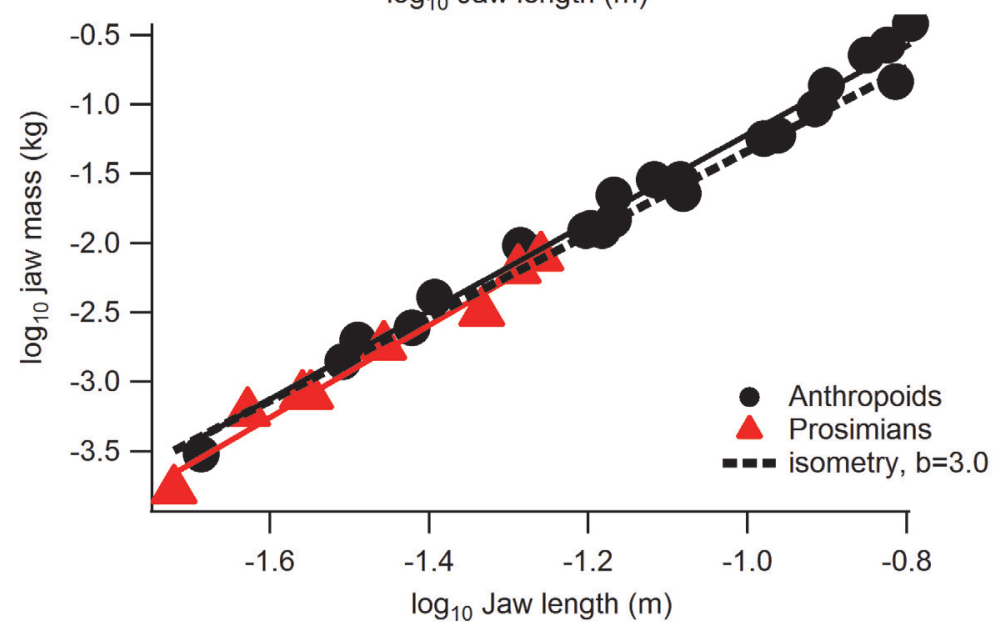

Figure 5. Bivariate $\log _{10}$ plots against jaw length of: $A$ ) distance from the weighted center of mass $\left(\mathrm{CoM}_{w}\right)$ to $A_{s c}$ and $\left.A_{m f} ; \mathrm{B}\right)$ distance from the $C o M_{w}$ to $A_{s c-o}$ and $\left.A_{m f-o} ; \mathrm{C}\right)$ jaw mass. PGLS lines are shown for anthropoids (anth.) and prosimians (prosim.). Lines of isometry are driven through the means of the data clusters. See Table 1 for abbreviations. 
A

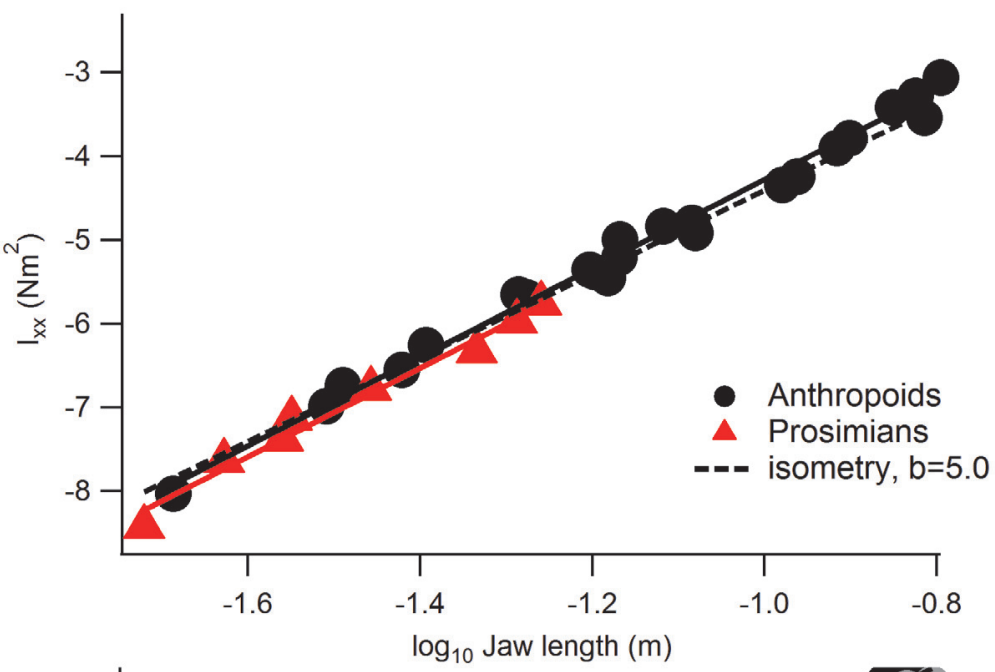

B

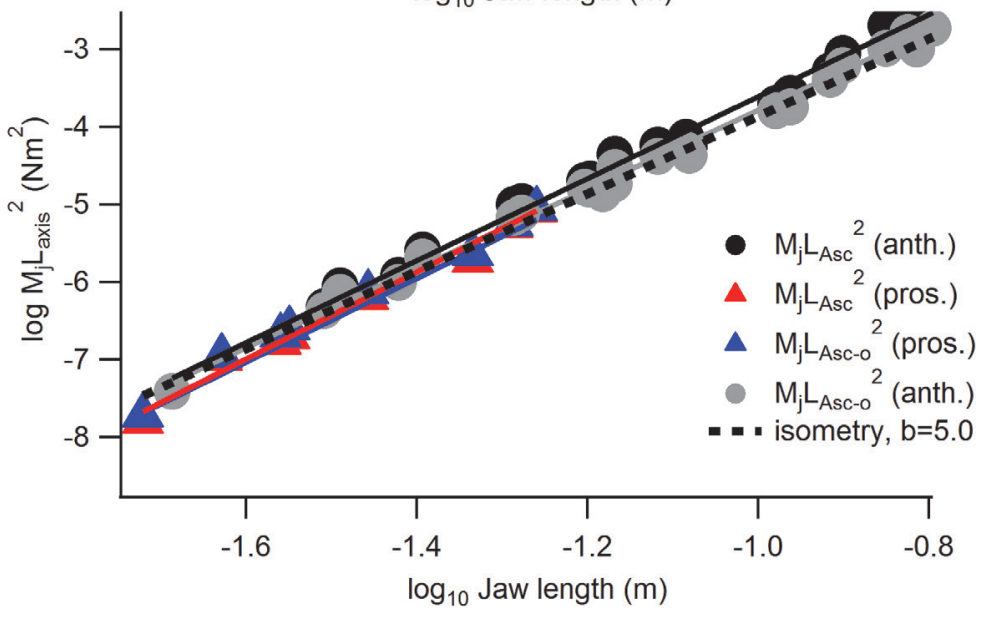

C

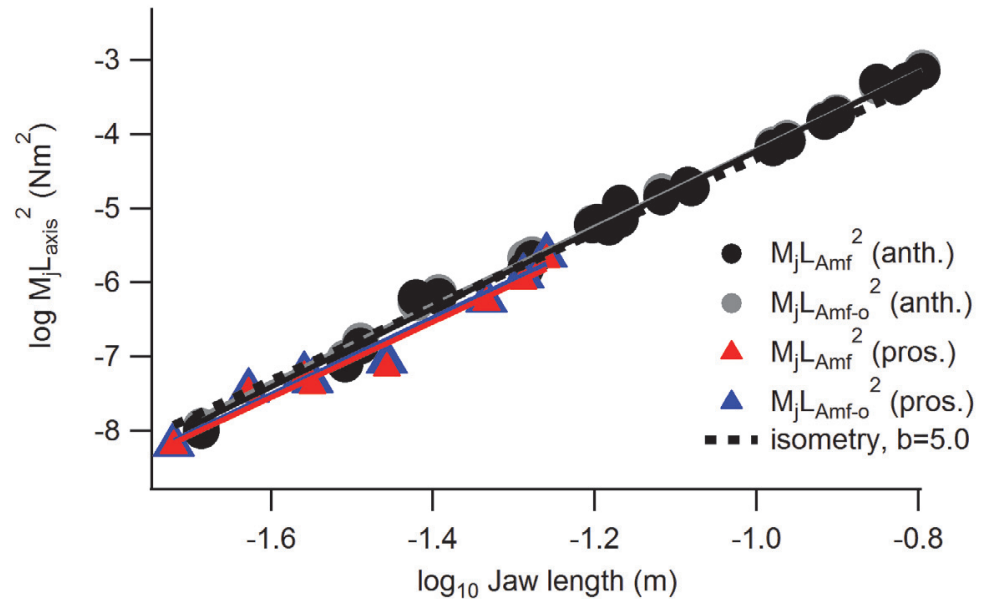

Figure 6. Bivariate $\log _{10}$ plots against jaw length of: A. $I_{x x}$, moment of inertia of mandible about a transverse axis through its center of mass; B. moment of inertia of mandible mass, concentrated at $\mathrm{CoM}_{w}$, about $A_{s c}$ and $A_{s c-o} ;$ C. moment of inertia of mandible mass, concentrated at $C o M_{w}$, about the $A_{m f}$ and $A_{m f-o}$. Phylogenetic least squares regression lines are 
shown for anthropoids and prosimians. Lines of isometry are driven through the means of the data clusters. See Table 1 for abbreviations.

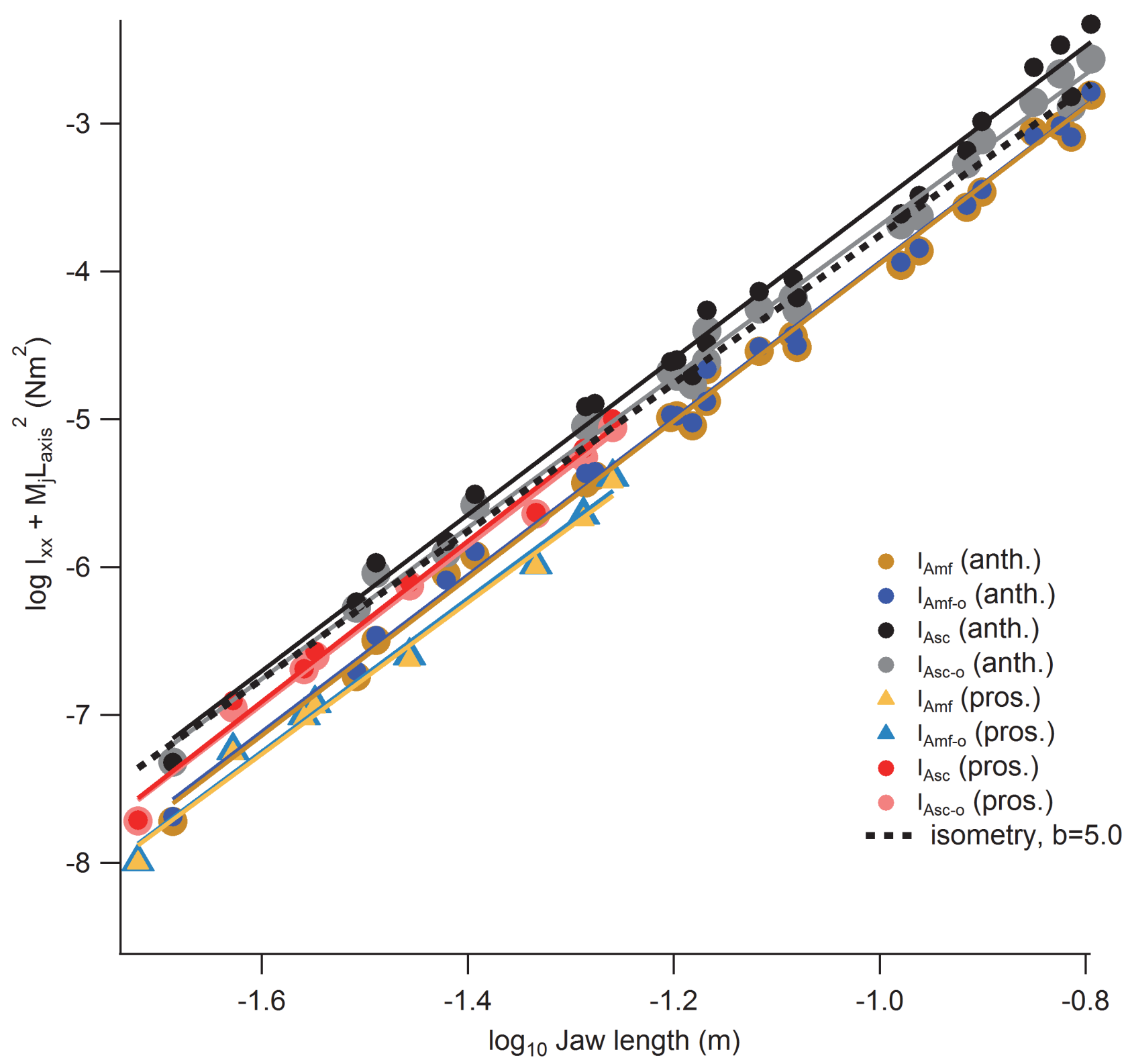

Figure 7. Bivariate $\log _{10}$ plot against jaw length of overall moment of inertia of the mandible about four transverse axes of rotation: $A_{s c}, A_{m f}, A_{s c-o}$, and $A_{m f-o}$. Line of isometry is driven through the means of the data clusters. See Table 1 for abbreviations. 


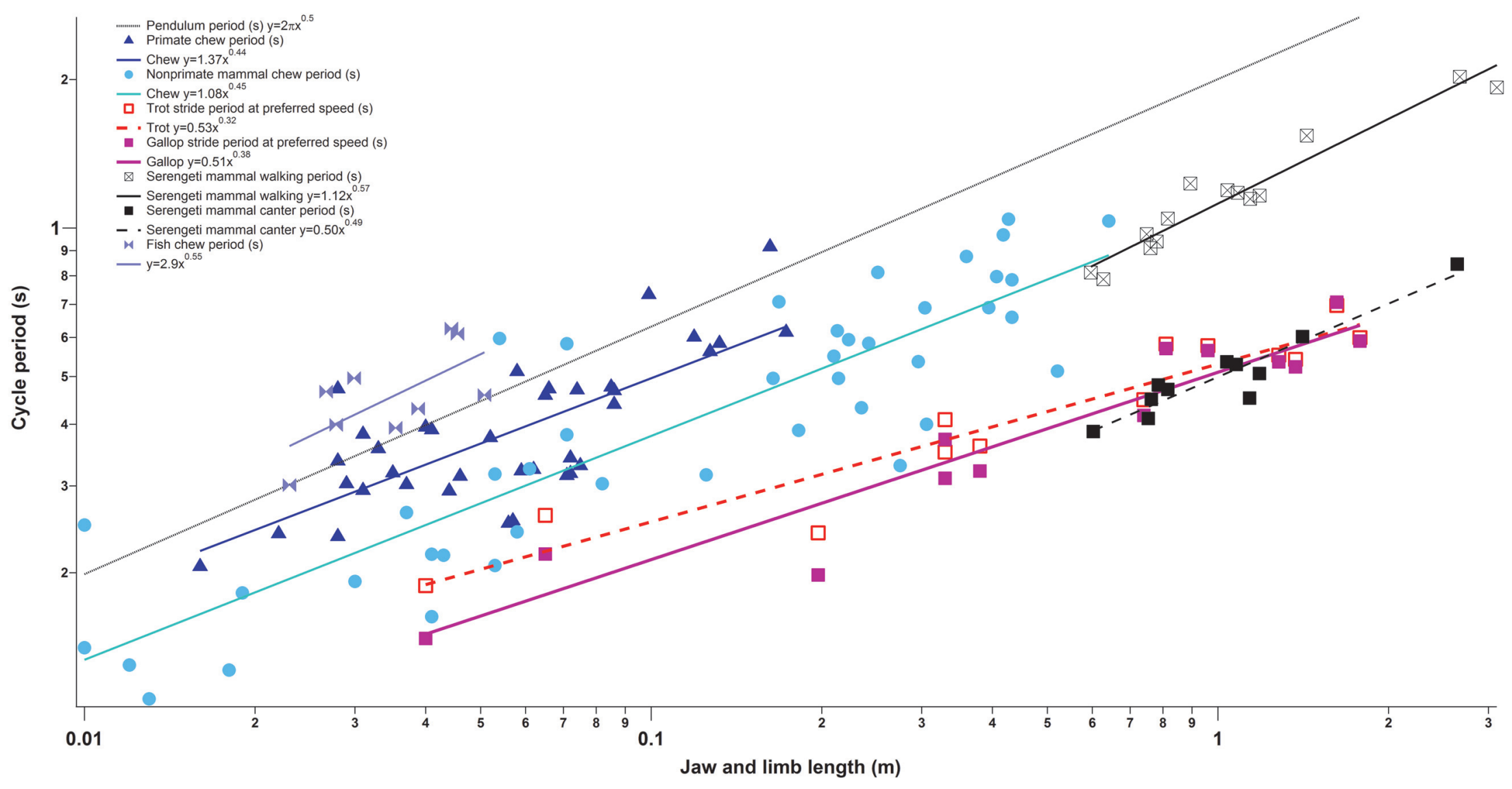

Figure 8. Scaling of chewing and locomotion cycle period versus jaw or limb length in mammals. Data on trot and gallop at preferred speed are from (Pontzer, 2007); Serengeti mammal data points were generated by digitizing points in Figure 13 of Pagel and Lutzoni (2002) and fitting lines with Pennycuick's exponents; primate chewing data and regression lines are from Vinyard and Ravosa (1998); nonprimate mammal data are from Gerstner et al. (in prep.); fish chewing data are from Gintof et al. (2010). Slopes from Pennycuick (1975) are exponential fits; fits from Pennycuick (1975) are ordinary least squares regressions with correlation coefficients > 0.9; all other slopes are standardized major axis. 